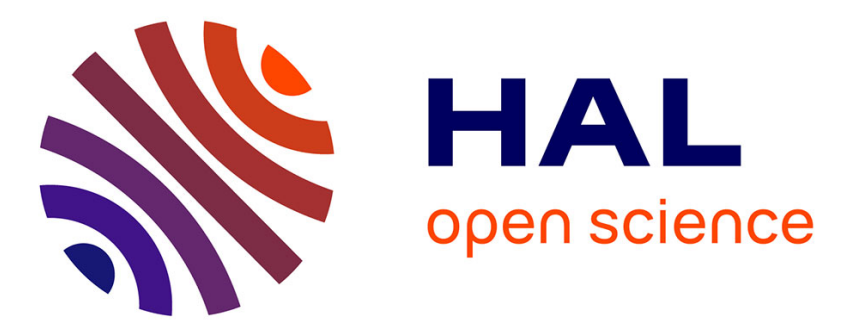

\title{
Modeling multivariate degradation processes with time-variant covariates and imperfect maintenance effects
}

Xiaolin Wang, Olivier Gaudoin, Laurent Doyen, Christophe Bérenguer, Min Xie

\section{To cite this version:}

Xiaolin Wang, Olivier Gaudoin, Laurent Doyen, Christophe Bérenguer, Min Xie. Modeling multivariate degradation processes with time-variant covariates and imperfect maintenance effects. Applied Stochastic Models in Business and Industry, 2021, 37 (3), pp.592-611. 10.1002/asmb.2600 . hal03138349

\section{HAL Id: hal-03138349 \\ https://hal.science/hal-03138349}

Submitted on 23 Feb 2021

HAL is a multi-disciplinary open access archive for the deposit and dissemination of scientific research documents, whether they are published or not. The documents may come from teaching and research institutions in France or abroad, or from public or private research centers.
L'archive ouverte pluridisciplinaire $\mathbf{H A L}$, est destinée au dépôt et à la diffusion de documents scientifiques de niveau recherche, publiés ou non, émanant des établissements d'enseignement et de recherche français ou étrangers, des laboratoires publics ou privés.

\section{(이)(\$)}

Distributed under a Creative Commons Attribution - NonCommercial - NoDerivatives 44.0 
DOI: $\mathrm{xxx} / \mathrm{xxxx}$

\title{
RESEARCH ARTICLE
}

\section{Modeling multivariate degradation processes with time-variant covariates and imperfect maintenance effects}

\author{
Xiaolin Wang ${ }^{1}$ | Olivier Gaudoin ${ }^{2}$ | Laurent Doyen ${ }^{2}$ | Christophe Bérenguer ${ }^{3}$ | Min Xie ${ }^{4}$
}

\footnotetext{
${ }^{1}$ Department of Logistics and Maritime Studies, The Hong Kong Polytechnic University, Kowloon, Hong Kong

${ }^{2}$ Univ. Grenoble Alpes, CNRS, Grenoble INP, LJK, Grenoble, France

${ }^{3}$ Univ. Grenoble Alpes, CNRS, Grenoble INP, GIPSA-lab, Grenoble, France

${ }^{4}$ Department of Systems Engineering and Engineering Management, City University of Hong Kong, Kowloon, Hong Kong
}

\section{Correspondence}

*Xiaolin Wang, Department of Logistics and Maritime Studies, The Hong Kong

Polytechnic University, Kowloon, Hong

Kong.

Email: xiao-lin.wang@polyu.edu.hk

\begin{abstract}
This article proposes two types of degradation models that are suitable for describing multivariate degrading systems subject to time-variant covariates and imperfect maintenance activities. A multivariate Wiener process is constructed as a baseline model, on top of which two types of models are developed to meaningfully characterize the time-variant covariates and imperfect maintenance effects. The underlying difference between the two models lies in the way of capturing the influences of covariates and maintenance: The first model reflects these impacts in the degradation rates/paths directly, whereas the second one describes the impacts by modifying the time scales governing the degradation processes. In each model, two particular imperfect maintenance models are presented, which differ in the extent of reduction in degradation level or virtual age. The two degradation models are then compared in certain special cases. The proposed multivariate degradation models pertain to complex industrial systems whose health deterioration can be characterized by multiple performance characteristics and can be altered or affected by maintenance activities and operating/environmental conditions.
\end{abstract}

\section{KEYWORDS:}

degradation path adjustment, imperfect maintenance, multivariate Wiener process, piece-wise constant covariates, time scale adjustment

\section{1 | INTRODUCTION}

\section{1 | Background and motivation}

Reliability and maintenance are crucial issues for many industrial systems, which have led to the development of associated theories and methodologies. Traditional reliability analysis relies heavily on failure data for choosing appropriate lifetime models, based on which various maintenance models are developed ${ }^{112}$. In recent years, due to the advances in low-cost sensoring and monitoring techniques, degradation models have become a prevailing alternative to traditional lifetime models ${ }^{3 / 4}$. The rationale of degradation-based reliability analysis is that aging failures of most systems can be attributed to the stochastic deterioration of some key performance characteristics (PCs) such as wear of machinery, capacity of batteries, and depth of tire tread ${ }^{[5}$. In this regard, degradation-based maintenance models can be developed by meaningfully characterizing the influence of maintenance activities on the underlying degradation processes; see, e.g., Kahle ${ }^{[6}$ and Mercier and Castro ${ }^{7}$, for recent references.

This article is interested in degradation and maintenance modeling for complex industrial systems. For such systems, there are three important features that should be considered in the modeling process: 
(i) Multiple performance characteristics. Most existing research on degradation modeling presumes that system deterioration can be well captured by only one PC. In reality, however, there are numerous industrial systems exhibiting degradation for multiple PCs, such as wear, crack, and vibration ${ }^{4}$. The degradation processes of a complex system are often statistically dependent, since they reflect the health condition of the same system. Another scenario leading to degradation dependency is that the same PC of a system is observed by multiple identical indicators (sensors) from different locations. In this scenario, the associated degradation processes shall be highly dependent.

(ii) Time-variant covariates. The operating and environmental conditions that systems operate in-referred to as covariates or explanatory variables in statistics — are auxiliary information that is closely related to system degradation processes. This is because operating and environmental conditions can induce internal stresses in a system that affect the rate or mode of system degradation -89 . Temperature, humidity, and operational profile are typical examples of such covariates. Quite often, the values of covariates are not constant all the time. We consider here that the covariates are piece-wise constant, which is a reasonable and mathematically tractable approximation of real scenarios.

(iii) Imperfect maintenance effects. Maintenance activities, either in a corrective or preventive manner, are indispensable for complex industrial systems, in terms of restoring a failed system to an operational status or mitigating the deterioration of a working system ${ }^{2}$. In particular, preventive maintenance (PM) programs are widely implemented to sustain continuous, cost-effective operations of industrial systems. Realistic maintenance activities are mostly imperfect in the sense that the system state after maintenance is between as-good-as-new and as-bad-as-old-the corresponding system states after perfect maintenance (or replacement) and minimal maintenance $\frac{1}{1}$.

The three features above are quite common yet essential for complex industrial systems; however, reported research that incorporates all of them into degradation modeling problems is surprisingly rare. This article intends to bridge this gap by developing two multivariate degradation models taking into account time-variant (more precisely, piece-wise constant) covariates and imperfect maintenance effects.

\section{2 | Related literature}

There are three streams of research closely related to our work - that is, multivariate degradation modeling, degradation-based imperfect maintenance modeling, and degradation modeling with time-variant (dynamic) covariates. As a side note, recent literature reviews on degradation and maintenance modeling can be found in van Noortwijk ${ }^{10}$, Ye and Xie ${ }^{3}$, Alaswad and

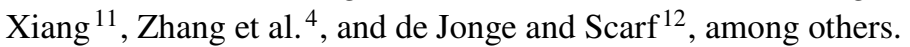

Reliability modeling, inference, and testing for systems/products subject to multiple degradation processes have attracted considerable attentions. In this stream of research, how to capture the dependency structure among individual degradation processes is a key issue. Many studies adopt copula functions for this purpose; see Sari et al. ${ }^{13}$, Pan et al. 14, Wang et al. ${ }^{15.16}$, Peng et al. ${ }^{17 \mid 18} 19$, Fang et al. ${ }^{20}$, Liu et al. ${ }^{[21}$, and Palayangoda and $\mathrm{Ng}^{22}$, for example. The prevalence of copula methods is largely attributed to its capability of separately modeling the dependency structure and univariate marginals; however, copula methods bear one drawback: Direct modeling of degradation increments by using a copula function does not preserve the infinite divisibility property 23 . On the other hand, some studies directly adopt either multivariate general path models $24 \mid 25$ or multivariate stochastic processes such as Wiener ${ }^{26|27| 28|29| 30 \mid 31}$ and gamma ${ }^{32|33|}$ to describe multivariate degradation processes. In addition, Mercier and $\mathrm{Pham}{ }^{34}$ and Mercier et al. ${ }^{35}$ introduce the so-called tri-variate reduction method to construct a bivariate gamma process. It is worth pointing out that most studies above focus on two PCs, resulting in various bivariate degradation processes. There are indeed a few exceptions that consider more than two PCs, including Hong et al. $\frac{36}{36}$, Si et al. $\frac{24}{3}$, Sun et al. $\frac{3738}{36}$,

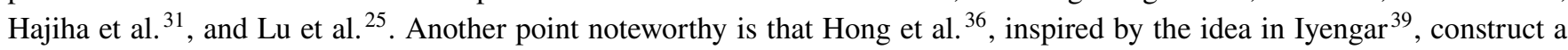
bivariate Wiener process from two univariate Wiener processes sharing a common noise. This idea of constructing multivariate degradation processes is adopted in the present article.

Moreover, maintenance modeling, inference, and planning for multivariate degrading systems are also prevailing research topics. Barker and Newby ${ }^{40}$ and Ahmadi ${ }^{41}$ study optimal inspection and replacement planning problems, respectively, for systems subject to a multivariate Wiener process. Mercier and Pham ${ }^{34}$ and Mercier et al. ${ }^{35}$ investigate similar problems for systems whose state is described by a bivariate Lévy process. Wu and Castro ${ }^{42}$ develop optimal PM policies for a system with a weighted

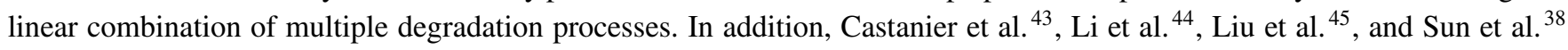
contribute to this field by studying condition-based replacement strategies for systems subject to multiple degradation processes. The above-summarized studies focus predominately on inspection or preventive replacement strategies. Imperfect maintenance, 
however, receives very few investigations in the context of multivariate degradation processes, though there are indeed some studies dealing with univariate degradation processes (see, e.g., Castanier et al. ${ }^{46}$; Mercier and Castro ${ }^{47}$; Zhang et al. ${ }^{48}$; Kahle ${ }^{6}$; Mercier and Castro ${ }^{7}$; Zhao et al. ${ }^{49}$; Salles et al. ${ }^{50}$ ). Basically, the extant degradation-based imperfect maintenance models, e.g., (virtual) age reduction ${ }^{6 / 47}$ and degradation level reduction ${ }^{6 / 7499}$, are created by mimicking the ideas of associated lifetime-based imperfect maintenance models. Nevertheless, developing imperfect maintenance models for multivariate degradation processes remains an open problem.

Furthermore, existing research on modeling multivariate degradation processes with time-variant/dynamic covariates is quite scarce. Singpurwalla ${ }^{8}$ provides a seminal overview on stochastic process-based failure models that are suitable to characterize dynamic environments. Meeker and Hong $\left[{ }^{[51}\right.$ and Hong et al. $\stackrel{[23}{ }$ discuss how to link big operating and environmental data as covariates to traditional reliability responses, including degradation signals. Peng et al. ${ }^{9}$ further summarize previous degradation-based reliability models that involve multiple degradation indicators and dynamic environments. Hong et al. ${ }^{52}$ and Xu et al. ${ }^{531}$ adopt a non-parametric model, precisely, shape-restricted splines, to estimate the influences of dynamic covariates on the degradation path. Peng et al. ${ }^{17}$ deal with multivariate degradation analysis of complex systems under dynamic operating and environmental conditions from a Bayesian perspective. Hajiha et al. ${ }^{31}$ investigate multivariate degradation modeling under dynamic operating conditions with both parametric and non-parametric approaches. Lu et al. ${ }^{25}$ propose a multivariate general path model with covariates and random effects to describe degradation data with multiple PCs. In addition, as we are dealing with piece-wise constant covariates, research on step-stress accelerated degradation test (ADT) is relevant and inspiring; see, e.g., Tseng and Wen ${ }^{54}$, Liao and Tseng ${ }^{55}$, Peng and Tseng ${ }^{56}$, Pan and Sun ${ }^{33}$, and Zhao et al. ${ }^{57}$. In particular, Pan and Sun ${ }^{33}$ study optimal stepstress ADT design for products subject to a bivariate gamma process. Generally speaking, the dominant approach to modeling covariates is to represent some parameters in the degradation model as functions of the covariates of interest, known as the link functions; see Ye and $\mathrm{Xie}^{\sqrt{3}}$ and Zhang et al. ${ }^{[4}$ for overviews.

To the best of our knowledge, no studies can be found to consider all of the three features mentioned earlier in degradation and maintenance modeling problems, despite its significance from both practical and academic perspectives.

\section{3 | Overview of this work}

This article contributes to the literature by providing two integrated frameworks of modeling multivariate degradation processes with imperfect maintenance effects and time-variant covariates. The multivariate Wiener process is adopted as a baseline model because it retains the independent increment and infinite divisibility properties. A method of constructing multivariate Wiener processes is first introduced, based on which two types of new models are further developed to incorporate the influences of imperfect PM and piece-wise constant covariates. In particular, the first model reflects these influences in the degradation rates/paths directly (referred to as the degradation path adjustment model), whereas the second one captures the influences by modifying the time scales governing the degradation processes (called the time scale adjustment model). Simulated degradation paths are sketched to illustrate the two types of degradation models. Comparisons between the two models in some special cases are also provided.

The focus of this article is on probabilistic modeling and simulation illustration. Inferential issues are beyond the scope of this article and, indeed, require further investigations. Section 2 formulates the piece-wise constant covariate process and introduces a new approach to constructing multivariate Wiener processes. The degradation path adjustment and time scale adjustment models are developed, respectively, in Sections 3 and 4 , and schematic illustrations are presented therein. Section 5 compares the proposed models in some special cases. Finally, Section 6 concludes this article and suggests some topics for future research.

\section{2 | MODEL FORMULATION}

In this section, we introduce the characterization of piece-wise constant covariates and the construction of multivariate Wiener processes, so as to form the basis for subsequent modeling efforts.

\section{1 | Piece-wise constant covariates}

Suppose there are $M$ covariates that have significant impacts on system degradation, and their values are piece-wise constant. Specifically, the covariates remain constant under a given operating and/or environmental stress, but might change their values 
(a) Covariate 1

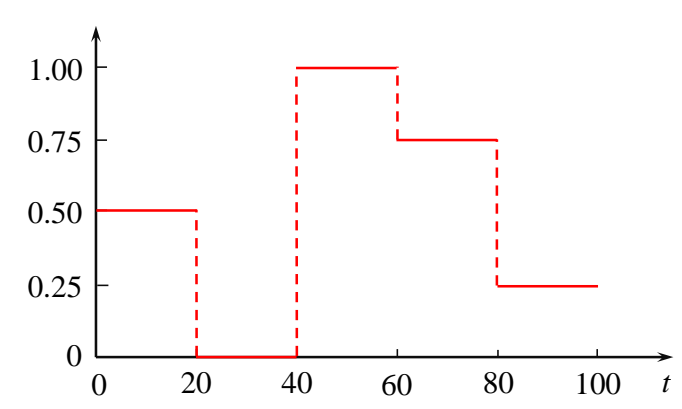

(b) Covariate 2

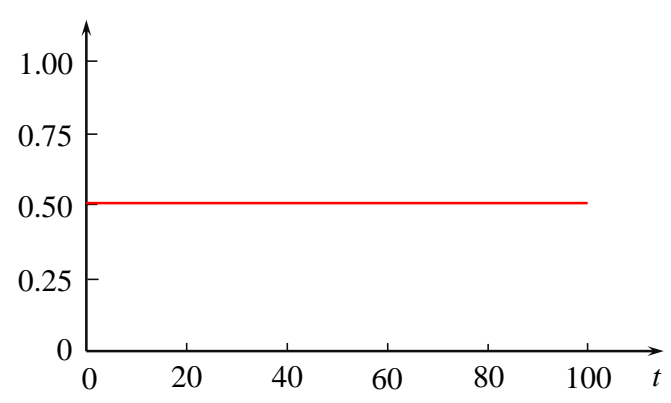

FIGURE 1 Schematic illustration of the evolution of two covariates $\left(\tau_{j}=20 \times j, j=1, \ldots, 5\right)$.

upon the shift of stress. Let $\tau_{j}, j=1,2, \ldots, J$, be the time point at which the operating/environmental stress shifts $\left(\tau_{0}=0\right)$, i.e., when at least one of the covariates changes its value. Let $s_{m, j}$ denote the constant value of the $m$ th covariate during the $j$ th time interval $\left[\tau_{j-1}, \tau_{j}\right), m=1,2, \ldots, M ; j=1,2, \ldots, J$. Note that the covariates might be subject to normalization or transformation;

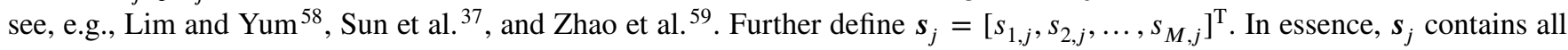
information on the $M$ covariates during $\left[\tau_{j-1}, \tau_{j}\right.$ ). Note also that the values of some covariates may not keep changing in every single period. In other words, not all corresponding elements in vectors $s_{j}$ and $\boldsymbol{s}_{j+l}(l=1,2, \ldots)$ are necessarily different, namely, there may exist some $m \in\{1,2, \ldots, M\}$ such that $s_{m, j}=s_{m, j+l}$.

In this manner, the piece-wise constant covariates at time $t$ can be fully described by

$$
\sum_{j=1}^{J} s_{j} \mathbf{1}_{\left[\tau_{j-1}, \tau_{j}\right)}(t)= \begin{cases}s_{1}, & 0 \leq t<\tau_{1}, \\ s_{2}, & \tau_{1} \leq t<\tau_{2}, \\ \vdots & \vdots \\ s_{J}, & \tau_{J-1} \leq t<\tau_{J},\end{cases}
$$

where $\mathbf{1}_{\mathcal{A}}(t)$ is an indicator function: if $t \in \mathcal{A}$ is true, then it is equal to $\mathbf{1}$; otherwise, it becomes $\mathbf{0}$. Further let $\mathbb{S}_{t}$ represent the entire history of the piece-wise constant covariate process from 0 to $t$.

Figure 1 illustrates the evolution of two covariates in five equal-length periods (i.e., $M=2, J=5$ ). We suppose that the shift of covariate value(s) occurs every 20 time units (i.e., $\tau_{j}=20 \times j, j=1, \ldots, 5$ ). The covariates are normalized so that their values are between zero and one. The value of covariate 1 keeps changing in every period $\left(s_{1,1}=0.50, s_{1,2}=0.00\right.$, $\left.s_{1,3}=1.00, s_{1,4}=0.75, s_{1,5}=0.25\right)$, whereas covariate 2 remains constant in all the five periods $\left(s_{2, j}=0.50, j=1, \ldots, 5\right)$. Possible examples of covariate 1 include workload, temperature, and humidity, whereas covariate 2 may be geographic location and system type, among others.

\section{2 | Multivariate degradation processes}

Consider a complex industrial system whose degradation is captured by $n$ PCs. Let $X_{i}(t), i=1,2, \ldots, n$, be the baseline degradation process of PC $i$ under some nominal covariates $s_{0}$. When actual covariate values differ from the nominal ones, the system's degradation processes can be affected. Note that in real applications, $X_{i}(t)$ might be subject to certain type of transformation (e.g., logarithm transformation) for data preprocessing purposes. As $X_{i}(t)$ 's describe the PCs of the same system, there should exist

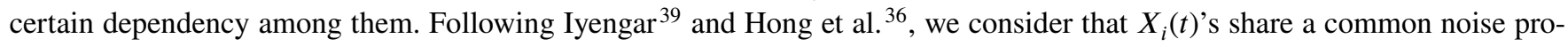
cess, but have their own noise processes as well. In essence, the common noise process characterizes the dependency among the $n$ degradation processes, whereas the distinct noise processes describe the uncertainty within individual degradation processes. To capture the time-varying volatility, we use Brownian motions to model these noises. Specifically, the baseline degradation process of PC $i$ is described by

$$
X_{i}(t)=\mu_{i} \Lambda(t)+\xi_{0} B^{(0)}(\Lambda(t))+\xi_{i} B^{(i)}(\Lambda(t)), i=1,2, \ldots, n,
$$



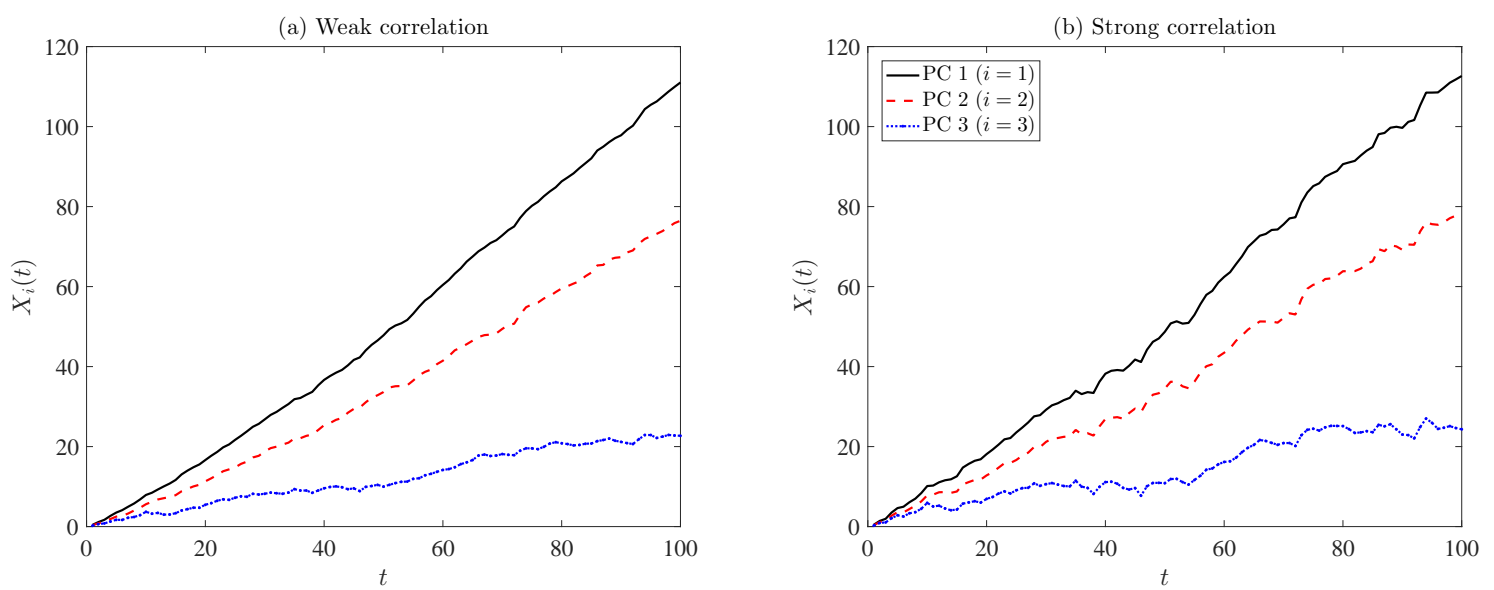

FIGURE 2 Simulated baseline degradation paths of three PCs in weak and strong correlation scenarios.

where $\mu_{i}$ is the drift parameter indicating the rate of degradation, $\xi_{0}$ and $\xi_{i}$ are diffusion parameters, $B^{(0)}(\cdot)$ and $B^{(i)}(\cdot)$ are independent standard Brownian motions, and $\Lambda(t)$ is a monotone increasing function capturing the nonlinearity in degradation processes ${ }^{26}$. A commonly used form of $\Lambda(t)$ is $\Lambda(t)=t^{\beta}$, where $\beta$ is a positive parameter. When $\Lambda(t)=t, X_{i}(t)$ has a linear mean path $\mu_{i} t$; otherwise, its mean path is non-linear.

It is easy to verify that $\mathrm{E}\left[X_{i}(t)\right]=\mu_{i} \Lambda(t), \operatorname{Var}\left(X_{i}(t)\right)=\left(\xi_{0}^{2}+\xi_{i}^{2}\right) \Lambda(t)$, and $\operatorname{Cov}\left(X_{i}(t), X_{i^{\prime}}(t)\right)=\xi_{0}^{2} \Lambda(t), i \neq i^{\prime}$. Thus, the correlation coefficient between any $X_{i}(t)$ and $X_{i^{\prime}}(t), i \neq i^{\prime}$, is given by

$$
\rho_{i, i^{\prime}}=\frac{\xi_{0}^{2}}{\sqrt{\left(\xi_{0}^{2}+\xi_{i}^{2}\right)\left(\xi_{0}^{2}+\xi_{i^{\prime}}^{2}\right)}} \in[0,1],
$$

which is independent of $t$. Note that when $\xi_{0} \rightarrow 0, X_{i}(t)$ and $X_{i^{\prime}}(t)$ tend to be independent; whereas when $\xi_{0} \gg \xi_{i}, i=1, \ldots, n$, the correlation between $X_{i}(t)$ and $X_{i^{\prime}}(t)$ would be positive and strong.

In this sense, the proposed multivariate Wiener process, constructed from multiple univariate Wiener processes sharing a common noise, is quite flexible in terms of describing various extents of dependency. This property is quite important for practical implementation: The operator may install multiple identical sensors (say, vibration sensors) in different locations of the same system, in which case the dependency among associated indicators shall be high; on the other hand, the dependency among indicators from different types of sensors (say, tension and vibration sensors) shall be relatively low. The two scenarios can be well characterized by sensibly estimating the values of drift and diffusion parameters.

Figure 2 illustrates three simulated baseline degradation paths in weak and strong correlation scenarios, respectively. The parameter setting used to generate this figure is as follows: $\mu_{1}=0.437, \mu_{2}=0.312, \mu_{3}=0.095, \xi_{1}=0.068, \xi_{2}=0.179$, $\xi_{3}=0.238$, and $\Lambda(t)=t^{1.2}$. In addition, $\xi_{0}$ is set to 0.25 in panel (a) and 0.75 in panel (b), representing weak and strong correlation scenarios, respectively. The degradation paths are simulated by the random walk approximation method; see Kahle et al.$\underline{60}$ for details on this method. Note that the baseline degradation paths in panel (a) will be used throughout Sections 3 and 4 for illustrative purposes.

Remark 1. An equivalent way of constructing model (2) is through the so-called $(n+1)$-variate reduction method ${ }^{3435}$. Suppose that we have $n+1$ independent univariate Wiener process: $Y_{0}(t)=\mu_{0} \Lambda(t)+\xi_{0} B^{(0)}(\Lambda(t))$ and $Y_{i}(t)=\left(\mu_{i}-\mu_{0}\right) \Lambda(t)+\xi_{i} B^{(i)}(\Lambda(t))$, $i=1,2, \ldots, n$. Then, $X_{i}(t)$ in (2) can be expressed as $X_{i}(t)=Y_{0}(t)+Y_{i}(t)$ for $i=1,2, \ldots, n$.

Remark 2. Let $\boldsymbol{X}(t)=\left[X_{1}(t), \ldots, X_{n}(t)\right]^{\mathrm{T}}$. The baseline degradation model in (2) is equivalent to a multivariate Wiener process 36 :

$$
\boldsymbol{X}(t)=\boldsymbol{\mu} \Lambda(t)+\boldsymbol{\Sigma}^{1 / 2} \boldsymbol{B}(\Lambda(t)),
$$

where $\boldsymbol{\mu}=\left[\mu_{1}, \ldots, \mu_{n}\right]^{\mathrm{T}}, \boldsymbol{B}(\cdot)$ is the standard $n$-dimensional Brownian motion, and $\boldsymbol{\Sigma}$ is the associated covariance matrix, which is positive definite. In particular, the $\left(i, i^{\prime}\right)$ th entry of $\boldsymbol{\Sigma}$ is $\sigma_{i, i}^{2}=\xi_{0}^{2}+\xi_{i}^{2}$ for $i=i^{\prime}$, and $\sigma_{i, i^{\prime}}^{2}=\xi_{0}^{2}$ for $i \neq i^{\prime}$. Thus, the correlation coefficient in (3) can be rewritten as $\rho_{i, i^{\prime}}=\sigma_{i, i^{\prime}}^{2} /\left(\sigma_{i, i} \sigma_{i^{\prime}, i^{\prime}}\right)$. Also, it is clear that $\boldsymbol{X}(t)$ follows a multivariate normal distribution, i.e., $\boldsymbol{X}(t) \sim \mathcal{N}(\boldsymbol{\mu} \Lambda(t), \boldsymbol{\Sigma} \Lambda(t))$. 
The baseline multivariate Wiener process will be used in Sections 3 and 4 to incorporate the influences of time-variant covariates and imperfect PM effects. It is well known that the Wiener process is not strictly monotone. This property is important for some self-healing cases such as the healing of cracks (caused by fatigue) and battery capacities. If a multivariate degradation process is believed to be monotonically increasing/decreasing, then one may resort to a multivariate gamma or inverse Gaussian process, possibly constructed via the $(n+1)$-variate reduction method.

\section{3 | DEGRADATION PATH ADJUSTMENT MODEL}

In this section, we consider the degradation path adjustment model which assumes that covariates and imperfect PM activities influence system degradation by directly adjusting the rates/paths of individual degradation processes.

\section{1 | Modeling the effect of piece-wise constant covariates}

Let $X_{i}\left(t \mid s_{j}\right)$ denote the degradation process of PC $i$ within $\left[\tau_{j-1}, \tau_{j}\right)$, during which the system operates under covariates $\boldsymbol{s}_{j}$, $i=1,2, \ldots, n ; j=1,2, \ldots, J$. Incorporating the covariates in an appropriate way requires a good understanding about how these factors affect the parameters of the degradation model ${ }^{8}$. In the degradation path adjustment model, we assume that the influences of piece-wise constant covariates on the individual degradation processes are reflected in the corresponding alterations of their drift parameters. Specifically, the drift parameters are modeled as link functions of the covariates. Let $\mu_{i}\left(\boldsymbol{s}_{j} ; \boldsymbol{\gamma}_{i}\right)$ be the drift parameter associated with $X_{i}\left(t \mid s_{j}\right)$, where $\gamma_{i}$ is the coefficient vector. Candidate forms of link functions include the linear relation, the Arrhenius relation, the exponential relation, and the power law relation $\frac{34}{4}$. Among them, the latter three can be unified in a common form of

$$
\mu_{i}\left(s_{j} ; \gamma_{i}\right)=\exp \left\{\gamma_{i, 0}+\sum_{m=1}^{M} \gamma_{i, m} s_{m, j}\right\}
$$

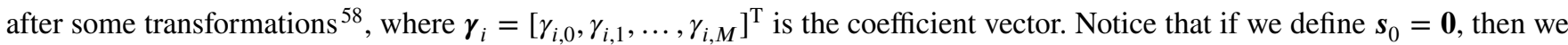
would have $\mu_{i}\left(s_{0} ; \gamma_{i}\right)=\exp \left\{\gamma_{i, 0}\right\}$, which can be treated as the baseline drift parameter $\mu_{i}$ in (2).

Denote $X_{i}\left(t ; \mathbb{S}_{t}\right)$ as the degradation process of PC $i$ subject to the piece-wise constant covariate process $\mathbb{S}_{t}$. Analogous to Liao and Tseng ${ }^{[5]}$ and Zhao et al. $\frac{57}{57}$ the relationships between $X_{i}\left(t ; \mathbb{S}_{t}\right)$ and $\left\{X_{i}\left(t \mid s_{j}\right), j=1,2, \ldots, J\right\}$ are modeled as follows. Under covariates $s_{1}$, it is clear that $X_{i}\left(t ; \mathbb{S}_{t}\right)=X_{i}\left(t \mid s_{1}\right)=\mu_{i}\left(s_{1} ; \gamma_{i}\right) \Lambda(t)+\xi_{0} B^{(0)}(\Lambda(t))+\xi_{i} B^{(i)}(\Lambda(t))$ for $t \in\left[0, \tau_{1}\right)$. Likewise, for $t \in\left[\tau_{1}, \tau_{2}\right)$ and under covariates $s_{2}$, the degradation process of PC $i$ becomes

$$
\begin{aligned}
X_{i}\left(t ; \mathbb{S}_{t}\right)= & X_{i}\left(\tau_{1} \mid s_{1}\right)+X_{i}\left(t \mid s_{2}\right)-X_{i}\left(\tau_{1} \mid s_{2}\right) \\
= & \mu_{i}\left(s_{1} ; \gamma_{i}\right) \Lambda\left(\tau_{1}\right)+\xi_{0} B^{(0)}\left(\Lambda\left(\tau_{1}\right)\right)+\xi_{i} B^{(i)}\left(\Lambda\left(\tau_{1}\right)\right)+\mu_{i}\left(s_{2} ; \gamma_{i}\right) \Lambda(t)+\xi_{0} B^{(0)}(\Lambda(t))+\xi_{i} B^{(i)}(\Lambda(t)) \\
& -\mu_{i}\left(s_{2} ; \gamma_{i}\right) \Lambda\left(\tau_{1}\right)-\xi_{0} B^{(0)}\left(\Lambda\left(\tau_{1}\right)\right)-\xi_{i} B^{(i)}\left(\Lambda\left(\tau_{1}\right)\right) \\
= & \mu_{i}\left(s_{1} ; \gamma_{i}\right) \Lambda\left(\tau_{1}\right)+\mu_{i}\left(s_{2} ; \gamma_{i}\right)\left(\Lambda(t)-\Lambda\left(\tau_{1}\right)\right)+\xi_{0} B^{(0)}(\Lambda(t))+\xi_{i} B^{(i)}(\Lambda(t)) .
\end{aligned}
$$

By doing so, an underlying assumption here is that the degradation process exhibits a memoryless property, which means that the rate of degradation — represented by the drift parameter $\mu_{i}\left(\boldsymbol{s}_{j} ; \boldsymbol{\gamma}_{i}\right)$-depends only on the current covariates $\boldsymbol{s}_{j}$ but not on the

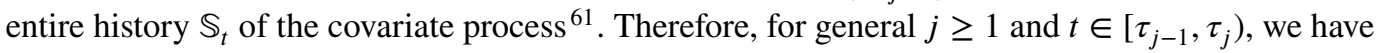

$$
\begin{aligned}
X_{i}\left(t ; \mathbb{S}_{t}\right) & =X_{i}\left(t \mid s_{j}\right)-X_{i}\left(\tau_{j-1} \mid s_{j}\right)+\sum_{l=1}^{j-1}\left(X_{i}\left(\tau_{l} \mid s_{l}\right)-X_{i}\left(\tau_{l-1} \mid s_{l}\right)\right) \\
& =\mu_{i}\left(s_{j} ; \gamma_{i}\right)\left(\Lambda(t)-\Lambda\left(\tau_{j-1}\right)\right)+\sum_{l=1}^{j-1} \mu_{i}\left(s_{l} ; \gamma_{i}\right)\left(\Lambda\left(\tau_{l}\right)-\Lambda\left(\tau_{l-1}\right)\right)+\xi_{0} B^{(0)}(\Lambda(t))+\xi_{i} B^{(i)}(\Lambda(t))
\end{aligned}
$$

By defining an adjusted mean degradation path as $d_{i}\left(t ; \mathbb{S}_{t}\right)=\mu_{i}\left(\boldsymbol{s}_{j} ; \boldsymbol{\gamma}_{i}\right)\left(\Lambda(t)-\Lambda\left(\tau_{j-1}\right)\right)+\sum_{l=1}^{j-1} \mu_{i}\left(\boldsymbol{s}_{l} ; \boldsymbol{\gamma}_{i}\right)\left(\Lambda\left(\tau_{l}\right)-\Lambda\left(\tau_{l-1}\right)\right)$, which is continuous over time, the degradation process of PC $i$ involving the piece-wise constant covariate process $\mathbb{S}_{t}$ can be rewritten as

$$
X_{i}\left(t ; \mathbb{S}_{t}\right)=d_{i}\left(t ; \mathbb{S}_{t}\right)+\xi_{0} B^{(0)}(\Lambda(t))+\xi_{i} B^{(i)}(\Lambda(t)), i=1, \ldots, n .
$$

Figure 3 shows the baseline and adjusted degradation paths of three PCs subject to piece-wise constant covariates under the degradation path adjustment model. We assume $\mu_{i}\left(s_{j} ; \gamma_{i}\right)=\mu_{i} \exp \left\{\gamma_{i, 1} s_{1, j}+\gamma_{i, 2} s_{2, j}\right\}$, and the associated parameters are set to $\gamma_{1,1}=0.156, \gamma_{1,2}=0.320, \gamma_{2,1}=0.535, \gamma_{2,2}=0.115, \gamma_{3,1}=0.093$, and $\gamma_{3,2}=-0.415$. The values of $\mu_{i}(i=1,2,3)$, 

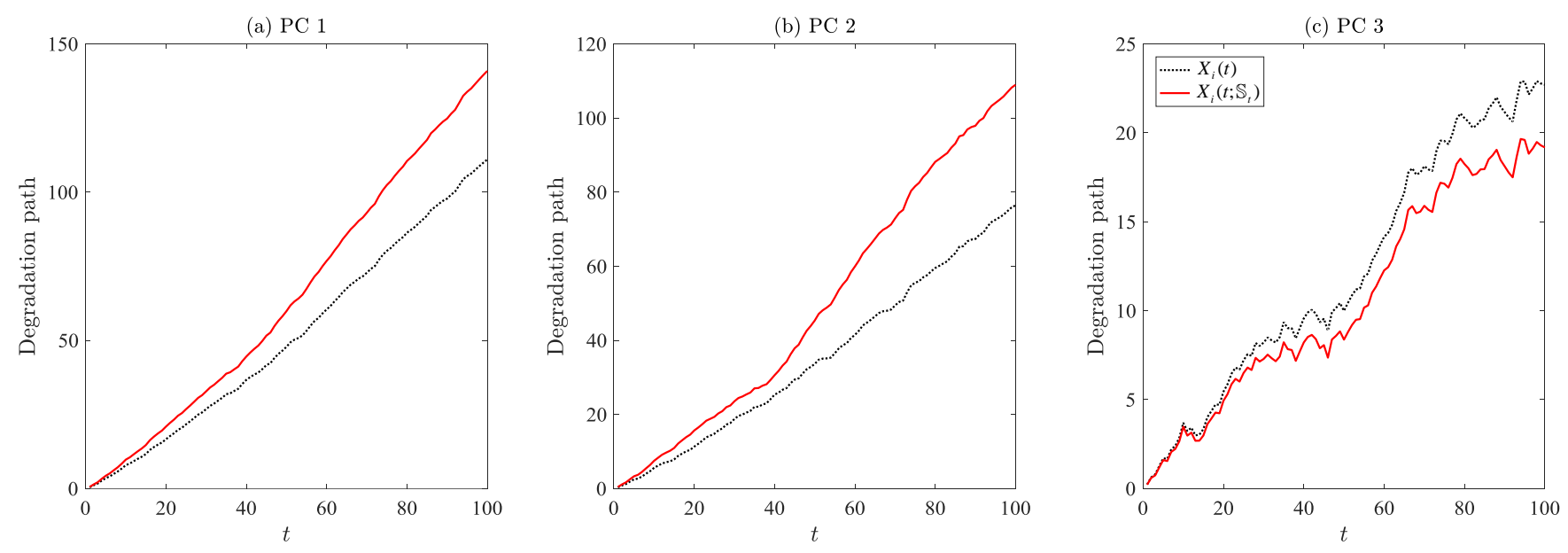

FIGURE 3 Baseline and adjusted degradation paths of three PCs with time-variant covariates under the degradation path adjustment model.

$s_{j}(j=1, \ldots, 5)$, and other parameters follow directly from Figures 1 and 2 In this figure, the baseline degradation paths (dotted lines) are the same as those in Figure 2 a). As can be seen, the piece-wise constant covariates could pull the baseline degradation paths up or down, depending on their positive or negative effects on the rates of degradation, and the rates change upon the shift of covariate values.

\subsection{Modeling the effect of imperfect PM actions}

Suppose that imperfect PM actions are performed at time instants $t_{1}, t_{2}, \ldots, t_{k}, \ldots$, with $t_{0}=0$. The maintenance instants $t_{k}$ and covariate shift instants $\tau_{j}$ are not necessarily identical for any $k$ and $j$. We further assume that if any failure occurs between two successive PM actions, then the system will be minimally repaired; after a minimal repair, the system is restored to an operational status, without any impact on its degradation processes.

In the degradation path adjustment model, the influence of imperfect PM activities is captured by the amount of degradation reduction before and after each PM activity. For convenience of notation, let $X_{i, k}=X_{i}\left(t_{k}^{-} ; \mathbb{S}_{t_{k}^{-}}\right)$represent the intrinsic (i.e., without maintenance) degradation level of PC $i$ at time $t_{k}^{-}, k=1,2, \ldots$; let $\eta_{i, k}$ be the associated degradation level immediately after the $k$ th PM activity. Define $\delta_{i, k} \in[0,1]$ as the degradation reduction factor of the $k$ th PM action for PC $i$. A larger value of $\delta_{i, k}$ corresponds to a higher maintenance efficiency. If $\delta_{i, k}=0$, then the maintenance effect is minimal; if $\delta_{i, k}=1$, then the maintenance effect is perfect; whereas $\delta_{i, k} \in(0,1)$ corresponds to an imperfect maintenance effect. In practice, for a specific PM action $k$, the degradation reduction factor $\delta_{i, k}$ for all $i$ might be correlated, as maintaining a system usually results in simultaneous degradation reduction for all of its PCs. A possible way of capturing this type of correlation is to model $\delta_{i, k}, i=1,2, \ldots, n$, as functions of the maintenance effort $e_{k}$, e.g., $\delta_{i, k}=\delta_{i}\left(e_{k}\right)$, where $\delta_{i}(\cdot)$ is a PC-specific function.

In principle, we might have the following two assumptions on the maintenance efficiency-following the ideas in Doyen and

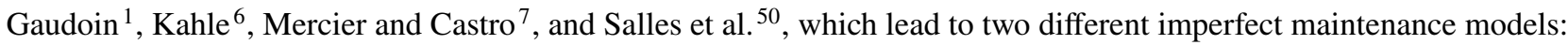

(i) $A R D_{1}$ model. The first model is called the Arithmetic Reduction of Degradation with memory one $\left(A R D_{1}\right)$. The idea is that an imperfect maintenance action only removes a proportion of the degradation accumulated since the last maintenance. As a result, the degradation level right after the $k$ th PM action can be formulated as

$$
\eta_{i, k}=\eta_{i, k-1}+\left(X_{i, k}-X_{i, k-1}\right)\left(1-\delta_{i, k}\right),
$$

which can be iteratively derived as $\eta_{i, k}=\sum_{l=1}^{k}\left(1-\delta_{i, l}\right)\left(X_{i, l}-X_{i, l-1}\right), k=1,2, \ldots$ Let $\tilde{X}_{i}\left(t ; \mathbb{S}_{t}\right)$ represent the degradation path of PC $i$ at $t \in\left[t_{k}, t_{k+1}\right)$, after the $k$ th PM action. Then, we have

$$
\begin{aligned}
\tilde{X}_{i}\left(t ; \mathbb{S}_{t}\right) & =\eta_{i, k}+X_{i}\left(t ; \mathbb{S}_{t}\right)-X_{i, k} \\
& =\sum_{l=1}^{k}\left(1-\delta_{i, l}\right)\left(X_{i, l}-X_{i, l-1}\right)+X_{i}\left(t ; \mathbb{S}_{t}\right)-X_{i, k}, k=1,2, \ldots,
\end{aligned}
$$



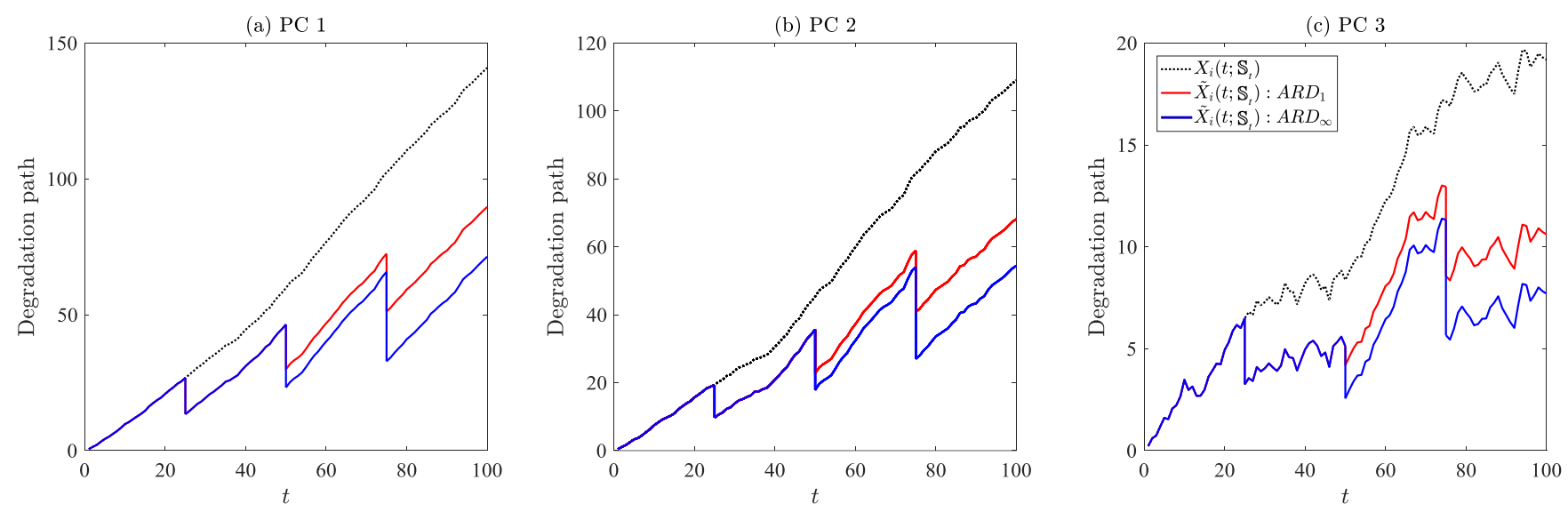

FIGURE 4 Degradation paths of three PCs involving time-variant covariates and imperfect PM actions under the degradation path adjustment model.

where $X_{i}\left(t ; \mathbb{S}_{t}\right)$ is given by $(8)$.

(ii) $A R D_{\infty}$ model. Another idea is that an imperfect maintenance action reduces the degradation of an amount proportional to the current degradation level. The corresponding model is called the Arithmetic Reduction of Degradation with infinite memory $\left(A R D_{\infty}\right)$. According to this assumption, one has

$$
\eta_{i, k}=\left(\eta_{i, k-1}+X_{i, k}-X_{i, k-1}\right)\left(1-\delta_{i, k}\right),
$$

which can be iteratively derived as $\eta_{i, k}=\sum_{l=1}^{k} \prod_{l^{\prime}=1}^{k+1-l}\left(1-\delta_{i, l^{\prime}}\right)\left(X_{i, l}-X_{i, l-1}\right), k=1,2, \ldots$. The corresponding degradation path of PC $i$ at $t \in\left[t_{k}, t_{k+1}\right)$, after the $k$ th PM, becomes

$$
\tilde{X}_{i}\left(t ; \mathbb{S}_{t}\right)=\sum_{l=1}^{k} \prod_{l^{\prime}=1}^{k+1-l}\left(1-\delta_{i, l^{\prime}}\right)\left(X_{i, l}-X_{i, l-1}\right)+X_{i}\left(t ; \mathbb{S}_{t}\right)-X_{i, k}, k=1,2, \ldots
$$

This way, $\tilde{X}_{i}\left(t ; \mathbb{S}_{t}\right)$ in $(10)$ or 12 —depending on which imperfect maintenance model is applied—is able to fully characterize the influences of both piece-wise constant covariates and imperfect PM activities on the degradation process. Figure 4 shows the simulated degradation paths of three PCs with both covariates and imperfect PM effects under the degradation path adjustment model. To generate this figure, we suppose that the PM interval is 25 time units and the degradation reduction factor is equal to 0.5 for all cases, namely, $t_{k}=25 \times k, \delta_{i, k}=0.5, i, k=1,2,3$. The dotted lines represent the degradation paths involving covariates only (i.e., the same as those red lines in Figure 3 ; the red and blue lines indicate the degradation paths with both covariates and imperfect maintenance (red: $A R D_{1}$ model; blue: $A R D_{\infty}$ model). As can be seen, between two successive PM actions, the degradation paths adjusted by imperfect maintenance are vertically parallel to the paths without maintenance. That is to say, if we move the red or blue paths upwards, then they would coincide with those dotted lines. This is because an imperfect PM action reduces the current degradation level to a lower level, and the degradation process then develops in the same rate as that without maintenance.

\section{3 | Further discussions}

It is worth mentioning that the proposed model can be reduced to a model with pure covariates or imperfect maintenance effects. More specifically, when $\mu_{i}\left(s_{j} ; \gamma_{i}\right)=\mu_{i}$ for all $j$, then the proposed model reduces to a model with only imperfect maintenance effect; when $\delta_{i, k}=0$ for all $k$, then there is only time-variant covariate effect; whereas when $\mu_{i}\left(s_{l} ; \gamma_{i}\right)=\mu_{i}$ and $\delta_{i, k}=0$ for all $j$ and $k$, then the proposed model simplifies to the original one in (2).

We now explore the probabilistic properties of the increments of individual degradation processes. Consider any time instant $t$ and time step $\Delta t$ that satisfy $t_{k} \leq t<t+\Delta t<t_{k+1}$. Define $\Delta \tilde{X}_{i}=\tilde{X}_{i}\left(t+\Delta t ; \mathbb{S}_{t+\Delta t}\right)-\tilde{X}_{i}\left(t ; \mathbb{S}_{t}\right), \Delta d_{i}=d_{i}\left(t+\Delta t ; \mathbb{S}_{t+\Delta t}\right)-d_{i}\left(t ; \mathbb{S}_{t}\right)$, and $\Delta \Lambda=\Lambda(t+\Delta t)-\Lambda(t)$. Then, for both $A R D_{1}$ and $A R D_{\infty}$ models, we have the following results.

Property 1. $\mathrm{E}\left[\Delta \tilde{X}_{i}\right]=\Delta d_{i}, \operatorname{Var}\left(\Delta \tilde{X}_{i}\right)=\left(\xi_{0}^{2}+\xi_{i}^{2}\right) \Delta \Lambda$, and $\operatorname{Cov}\left(\Delta \tilde{X}_{i}, \Delta \tilde{X}_{i^{\prime}}\right)=\xi_{0}^{2} \Delta \Lambda$ for $i \neq i^{\prime}$. 
All proofs in this article can be found in the Appendix. Based on the results in Property 1 , the correlation coefficient between any $\Delta \tilde{X}_{i}$ and $\Delta \tilde{X}_{i^{\prime}}, i \neq i^{\prime}$, can be derived as $\tilde{\rho}_{i, i^{\prime}}=\xi_{0}^{2} / \sqrt{\left(\xi_{0}^{2}+\xi_{i}^{2}\right)\left(\xi_{0}^{2}+\xi_{i^{\prime}}^{2}\right)}$, which is exactly the same as $\rho_{i, i^{\prime}}$ in (3). This implies that the incorporation of covariates and imperfect maintenance effects via the degradation path adjustment model does not change the dependency structure among individual degradation processes; also, the infinite divisibility property is well preserved. Let $\Delta \tilde{\boldsymbol{X}}=\left[\Delta \tilde{X}_{1}, \ldots, \Delta \tilde{X}_{n}\right]^{\mathrm{T}}$ and $\Delta \tilde{\boldsymbol{d}}=\left[\Delta \tilde{d}_{1}, \ldots, \Delta \tilde{d}_{n}\right]^{\mathrm{T}}$. Then, for any $t_{k} \leq t \leq t+\Delta t \leq t_{k+1}$, the increment of the multivariate degradation process, i.e., $\Delta \tilde{\boldsymbol{X}}$, follows a multivariate normal distribution with mean $\Delta \tilde{\boldsymbol{d}}$ and covariance matrix $\boldsymbol{\Sigma} \Delta \Lambda$, where $\boldsymbol{\Sigma}$ is the same as that in Remark 2 This result is helpful for parameter estimation of the model.

Remark 3. In addition to degradation reduction, an imperfect maintenance action might have another key effect: Practical obser-

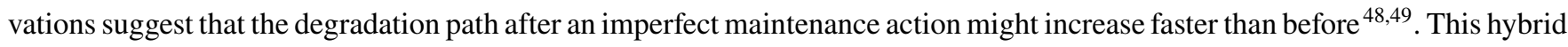
effect of imperfect maintenance can be captured by a degradation-rate increase factor $\phi_{k}\left(\phi_{k} \geq 1, k=1,2, \ldots\right)$, with $\phi_{0}=1$. After the $k$ th PM action, the degradation path can be modified to $\tilde{X}_{i}\left(t ; \mathbb{S}_{t}\right)=\eta_{i, k}+\phi_{k}\left[d_{i}\left(t ; \mathbb{S}_{t}\right)-d_{i}\left(t_{k} ; \mathbb{S}_{t}\right)\right]+\xi_{0}\left[B^{(0)}(\Lambda(t))-\right.$ $\left.B^{(0)}\left(\Lambda\left(t_{k}\right)\right)\right]+\xi_{i}\left[B^{(i)}(\Lambda(t))-B^{(i)}\left(\Lambda\left(t_{k}\right)\right)\right], t \in\left[t_{k}, t_{k+1}\right), k=1,2, \ldots$ Nevertheless, the modified degradation path $\tilde{X}_{i}\left(t ; \mathbb{S}_{t}\right)$ should be fed back to $(7)$ to revise $X_{i}\left(t ; \mathbb{S}_{t}\right)$, which complicate the problem. We thus leave this issue for future research.

\section{4 | TIME SCALE ADJUSTMENT MODEL}

In this section, we introduce the time scale adjustment model. The principle of this model is to consider that covariates and imperfect maintenance activities modify the time scales governing the degradation processes.

\section{1 | Modeling the effect of piece-wise constant covariates}

Again, let $X_{i}\left(t \mid s_{j}\right)$ represent the $i$ th degradation process under covariates $\boldsymbol{s}_{j}$, during the time interval $\left[\tau_{j-1}, \tau_{j}\right)$. In the time scale adjustment model, the influences of piece-wise constant covariates on system degradation is characterized by the modification of time scales. As the system is supposed to operate under different covariates one period after another, actual (chronological) operating time is not capable to reflect system degradation under different covariate levels. ${ }^{62}$ In order to facilitate degradation correspondence in different regimes, we introduce the concept of equivalent operating time. The rationale of this concept is that for a degradation process exposed under a specific covariate level for a certain time period, there exists an equivalent operating time for this process under another covariate level. In particular, the existence of such an equivalent operating time is obvious when the covariate of interest is usage rate; while for other types of covariates, the cumulative exposure model ${ }^{61}$ guarantees the existence of an equivalent operating time.

Let $\theta_{i, j}$ represent the equivalent operating time of degradation process $i$ when the system starts operating under covariates $\boldsymbol{s}_{j}$ $\left(\theta_{i, 1}=0\right)$. With the concept of equivalent operating time, exposing PC $i$ under covariates $s_{j}$ for $\theta_{i, j}$ time units is equivalent to exposing it under covariates $s_{j-1}$ for $\tau_{j-1}-\tau_{j-2}+\theta_{i, j-1}$ time units $\frac{5456}{}$; see Figure 5 for demonstration. From the degradation perspective, this statement corresponds to

$$
X_{i}\left(\theta_{i, j} \mid s_{j}\right)=X_{i}\left(\tau_{j-1}-\tau_{j-2}+\theta_{i, j-1} \mid s_{j-1}\right), j \geq 2 .
$$

In this manner, during $\left[\tau_{j-1}, \tau_{j}\right)$ and under covariates $\boldsymbol{s}_{j}$, the $i$ th degradation process at time $t$ can be expressed in terms of $\theta_{i, j}$, as

$$
\begin{aligned}
& X_{i}\left(t ; \mathbb{S}_{t}\right)=X_{i}\left(t \mid s_{1}\right), j=1 ; \\
& X_{i}\left(t ; \mathbb{S}_{t}\right)=X_{i}\left(t-\tau_{j-1}+\theta_{i, j} \mid s_{j}\right), j \geq 1 .
\end{aligned}
$$

In order to obtain an explicit expression of $X_{i}\left(t ; \mathbb{S}_{t}\right)$, we need to specify a detailed formulation for $X_{i}\left(t \mid \boldsymbol{s}_{j}\right)$. Following Peng

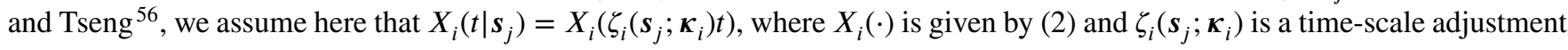
function with $\boldsymbol{\kappa}_{i}$ being its coefficient vector. In essence, if $\zeta_{i}\left(s_{j} ; \boldsymbol{\kappa}_{i}\right)>1$ (resp. $<1$ ), then degradation process $i$ is accelerated (resp. decelerated) by the stresses induced by covariates. A candidate form of the time-scale adjustment function is $\zeta_{i}\left(\boldsymbol{s}_{j} ; \boldsymbol{\kappa}_{i}\right)=$ $\exp \left\{\sum_{m=1}^{M} \kappa_{i, m} s_{m, j}\right\}$, where $\boldsymbol{\kappa}_{i}=\left[\kappa_{i, 1}, \kappa_{i, 2}, \ldots, \kappa_{i, M}\right]^{\mathrm{T}}$. According to this form, we have $\zeta_{i}\left(\boldsymbol{s}_{0} ; \boldsymbol{\kappa}_{i}\right)=1$ for $\boldsymbol{s}_{0}=\mathbf{0}$, namely, the time scale remains unchanged under nominal covariates $s_{0}$ and the corresponding degradation process reduces to the baseline model in (2).

Substituting $X_{i}\left(t \mid s_{j}\right)=X_{i}\left(\zeta_{i}\left(\boldsymbol{s}_{j} ; \boldsymbol{\kappa}_{i}\right) t\right)$ into $[13$ yields

$$
\zeta_{i}\left(\boldsymbol{s}_{j} ; \boldsymbol{\kappa}_{i}\right) \theta_{i, j}=\zeta_{i}\left(\boldsymbol{s}_{j-1} ; \boldsymbol{\kappa}_{i}\right)\left(\tau_{j-1}-\tau_{j-2}+\theta_{i, j-1}\right), j \geq 2,
$$




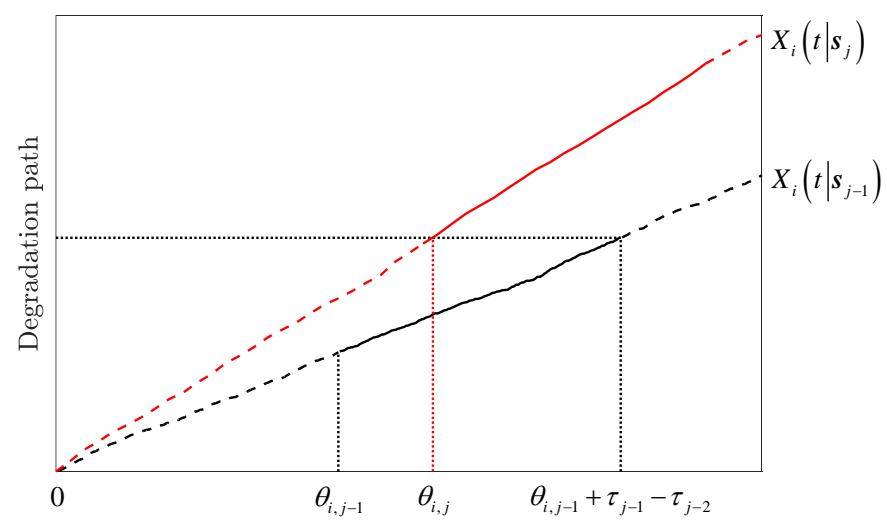

FIGURE 5 Schematic illustration of the concept of equivalent operating time.

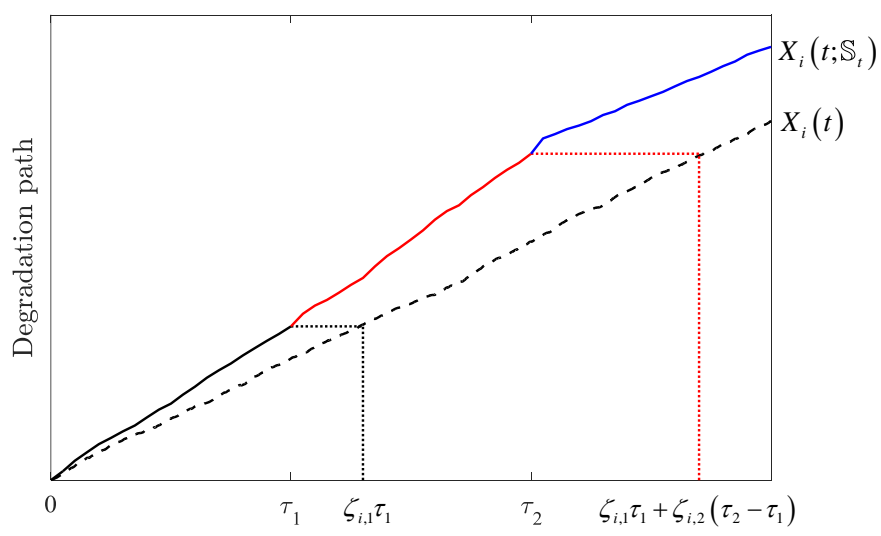

FIGURE 6 Conversion to equivalent operating time under nominal covariates $\boldsymbol{s}_{0}\left(\zeta_{i, j}\right.$ stands for $\left.\zeta_{i}\left(\boldsymbol{s}_{j} ; \boldsymbol{\kappa}_{i}\right), j=1,2\right)$.

which can be iteratively derived as

$$
\zeta_{i}\left(\boldsymbol{s}_{j} ; \boldsymbol{\kappa}_{i}\right) \theta_{i, j}=\sum_{l=2}^{j} \zeta_{i}\left(\boldsymbol{s}_{l-1} ; \boldsymbol{\kappa}_{i}\right)\left(\tau_{l-1}-\tau_{l-2}\right), j \geq 2 .
$$

This way, we can convert the actual operating time periods under all covariate levels $\boldsymbol{s}_{j}$, i.e., $\tau_{j}-\tau_{j-1}, j=1,2, \ldots, J$, to the corresponding equivalent operating time periods under nominal covariates $s_{0}$, i.e., $\zeta_{i}\left(s_{j} ; \boldsymbol{\kappa}_{i}\right)\left(\tau_{j}-\tau_{j-1}\right)$. For illustrative purposes, Figure 6 shows that the degradation amount of process $i$ accumulated under covariate $s_{1}$ for $\tau_{1}$ time units is equivalent to that under covariate $\boldsymbol{s}_{0}$ for $\zeta_{i}\left(\boldsymbol{s}_{1} ; \boldsymbol{\kappa}_{i}\right) \tau_{1}$ time units; similarly, the degradation amount at $\tau_{2}$ is equivalent to that accumulated under covariate $\boldsymbol{s}_{0}$ for $\zeta_{i}\left(\boldsymbol{s}_{1} ; \boldsymbol{\kappa}_{i}\right) \tau_{1}+\zeta_{i}\left(\boldsymbol{s}_{2} ; \boldsymbol{\kappa}_{i}\right)\left(\tau_{2}-\tau_{1}\right)$ time units.

By introducing an adjusted time scale $\omega_{i}\left(t ; \mathbb{S}_{t}\right)$ as

$$
\omega_{i}\left(t ; \mathbb{S}_{t}\right)= \begin{cases}\zeta_{i}\left(\boldsymbol{s}_{1} ; \boldsymbol{\kappa}_{i}\right) t, & 0 \leq t<\tau_{1}, \\ \zeta_{i}\left(\boldsymbol{s}_{2} ; \boldsymbol{\kappa}_{i}\right)\left(t-\tau_{1}\right)+\zeta_{i}\left(\boldsymbol{s}_{1} ; \boldsymbol{\kappa}_{i}\right) \tau_{1}, & \tau_{1} \leq t<\tau_{2}, \\ \vdots & \vdots \\ \zeta_{i}\left(\boldsymbol{s}_{j} ; \boldsymbol{\kappa}_{i}\right)\left(t-\tau_{j-1}\right)+\sum_{l=2}^{j} \zeta_{i}\left(\boldsymbol{s}_{l-1} ; \boldsymbol{\kappa}_{i}\right)\left(\tau_{l-1}-\tau_{l-2}\right), & \tau_{j-1} \leq t<\tau_{j}, \\ \vdots & \vdots,\end{cases}
$$

the $i$ th degradation process with the piece-wise constant covariate process $\mathbb{S}_{t}$ can be reformulated as

$$
X_{i}\left(t ; \mathbb{S}_{t}\right)=\mu_{i} \Lambda\left(\omega_{i}\left(t ; \mathbb{S}_{t}\right)\right)+\xi_{0} B^{(0)}\left(\Lambda\left(\omega_{i}\left(t ; \mathbb{S}_{t}\right)\right)\right)+\xi_{i} B^{(i)}\left(\Lambda\left(\omega_{i}\left(t ; \mathbb{S}_{t}\right)\right)\right), i=1,2, \ldots, n .
$$


(a) $\mathrm{PC} 1$

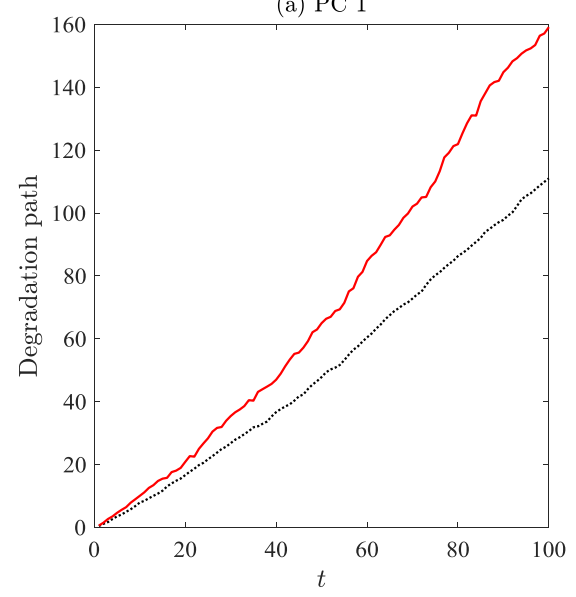

(b) $\mathrm{PC} 2$

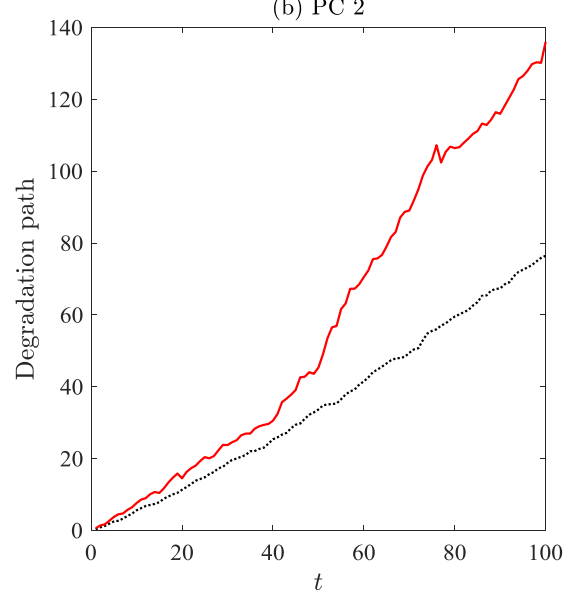

(c) $\mathrm{PC} 3$

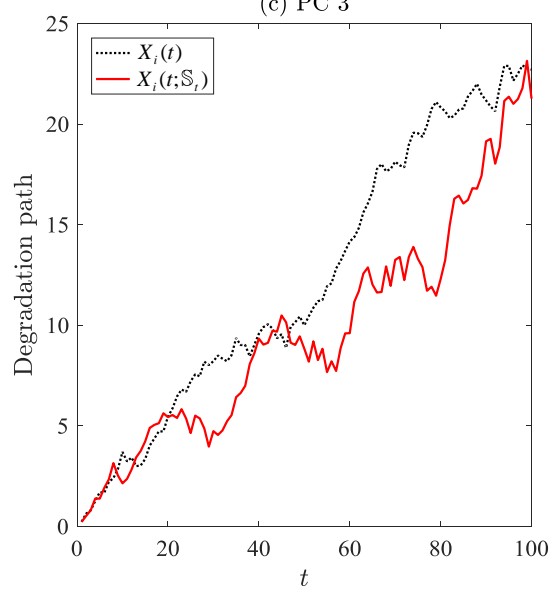

FIGURE 7 Baseline and adjusted degradation paths of three PCs with time-variant covariates under the time scale adjustment model.

Notice that $\omega_{i}\left(t ; \mathbb{S}_{t}\right)$ is a piece-wise, continuous function. Figure 7 shows the baseline and adjusted degradation paths of three PCs subject to piece-wise constant covariates under the time scale adjustment model. We assume $\zeta_{i}\left(\boldsymbol{s}_{j} ; \boldsymbol{\kappa}_{i}\right)=\exp \left\{\kappa_{i, 1} s_{1, j}+\right.$ $\left.\kappa_{i, 2} s_{2, j}\right\}$, and its parameters are set to $\kappa_{1,1}=0.156, \kappa_{1,2}=0.320, \kappa_{2,1}=0.535, \kappa_{2,2}=0.115, \kappa_{3,1}=0.093$, and $\kappa_{3,2}=-0.415$. The values of other parameters follow directly from Figures 1 and 2 In this figure, the baseline degradation paths (dotted lines) are again the same as those in Figure 2 a). Unlike the degradation path adjustment model in which covariates lead to either increase or decrease in the degradation rates, the time scale adjustment model reflects the influence of covariates in the stretching of the time scales. As the Wiener process is non-monotone, the adjusted degradation paths may intersect with the baseline paths. This explains the phenomenon that the two curves are crossing in Figure 7 (c).

\section{2 | Modeling the effect of imperfect PM actions}

Suppose again that imperfect PM actions are performed at time instants $t_{1}, t_{2}, \ldots, t_{k}, \ldots$. For convenience of notation, let $\omega_{i, k}$ represent the equivalent operating time of degradation process $i$ at time $t_{k}^{-}$(say, under certain $s_{j}$ and $t_{k} \in\left[\tau_{j-1}, \tau_{j}\right.$ )). The value of $\omega_{i, k}$ can be easily obtained by substituting $t_{k}^{-}$into (17). Further let $v_{i, k}$ denote the virtual age of degradation process $i$ immediately after the $k$ th PM action. The virtual age concept is initially introduced by Kijima ${ }^{63}$ in the context of recurrent events, and then adapted to maintenance modeling of deteriorating systems ${ }^{677}$; see Finkelstein and $\mathrm{Cha}^{62}$ for a recent discussion on virtual age in reliability context. In the time scale adjustment model, the effect of an imperfect PM activity is described by the amount of age reduction between $\omega_{i, k}$ and $v_{i, k}$. By mimicking the virtual age reduction ideas in Doyen and Gaudoin ${ }^{1}$, Mercier and Castro $\frac{747}{}$, and Kahle ${ }^{6}$, below we introduce two imperfect maintenance models - the Arithmetic Reduction of Age with memory one $\left(A R A_{1}\right)$ and Arithmetic Reduction of Age with infinite memory $\left(A R A_{\infty}\right)$.

(i) $A R A_{1}$ model. This model assumes that an imperfect maintenance action only removes a proportion of the age accumulated since the last maintenance. As a result, the virtual age of degradation process $i$ right after the $k$ th PM action is given by

$$
v_{i, k}=v_{i, k-1}+\left(\omega_{i, k}-\omega_{i, k-1}\right)\left(1-\delta_{i, k}\right),
$$

which can be iteratively derived as $v_{i, k}=\sum_{l=1}^{k}\left(1-\delta_{i, l}\right)\left(\omega_{i, l}-\omega_{i, l-1}\right), k=1,2, \ldots$.

Let $\tilde{\omega}_{i}\left(t ; \mathbb{S}_{t}\right)$ represent the virtual time scale of degradation process $i$ at $t \in\left[t_{k}, t_{k+1}\right)$ after the $k$ th imperfect PM action. Then, $\tilde{\omega}_{i}\left(t ; \mathbb{S}_{t}\right)$ evolves as follows:

$$
\begin{aligned}
\tilde{\omega}_{i}\left(t ; \mathbb{S}_{t}\right) & =v_{i, k}+\omega_{i}\left(t ; \mathbb{S}_{t}\right)-\omega_{i, k} \\
& =\sum_{l=1}^{k}\left(1-\delta_{i, l}\right)\left(\omega_{i, l}-\omega_{i, l-1}\right)+\omega_{i}\left(t ; \mathbb{S}_{t}\right)-\omega_{i, k}, k=1,2, \ldots,
\end{aligned}
$$

where $\omega_{i}\left(t ; \mathbb{S}_{t}\right)$ is given by $[17)$. 
(a) $\mathrm{PC} 1$

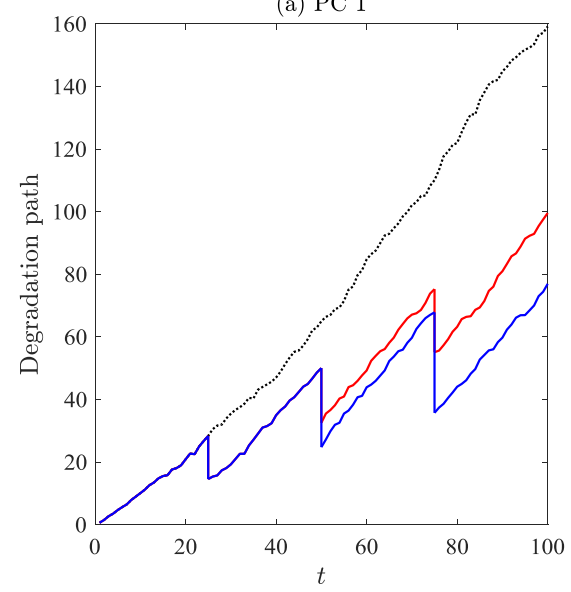

(b) PC 2

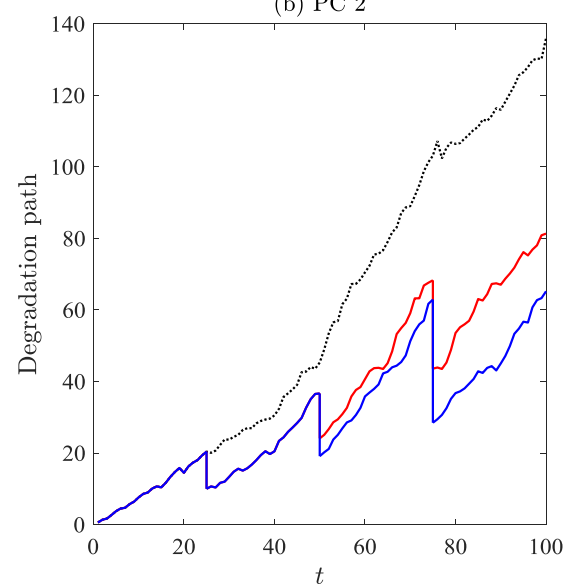

(c) $\mathrm{PC} 3$

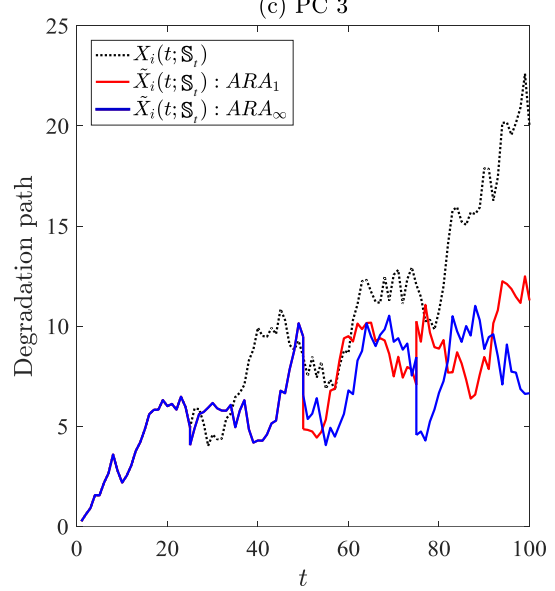

FIGURE 8 Degradation paths of three PCs involving time-variant covariates and imperfect PM effects under the time scale adjustment model.

(ii) $A R A_{\infty}$ model. The assumption of this model is that an imperfect maintenance action reduces the virtual age of an amount proportional to its age just before maintenance. This leads to

$$
v_{i, k}=\left(v_{i, k-1}+\omega_{i, k}-\omega_{i, k-1}\right)\left(1-\delta_{i, k}\right),
$$

which is equivalent to $v_{i, k}=\sum_{l=1}^{k} \prod_{l^{\prime}=1}^{k+1-l}\left(1-\delta_{i, l^{\prime}}\right)\left(\omega_{i, l}-\omega_{i, l-1}\right), k=1,2, \ldots$ In this case, the virtual time scale $\tilde{\omega}_{i}\left(t ; \mathbb{S}_{t}\right)$ at $t \in\left[t_{k}, t_{k+1}\right)$ evolves as follows:

$$
\tilde{\omega}_{i}\left(t ; \mathbb{S}_{t}\right)=\sum_{l=1}^{k} \prod_{l^{\prime}=1}^{k+1-l}\left(1-\delta_{i, l^{\prime}}\right)\left(\omega_{i, l}-\omega_{i, l-1}\right)+\omega_{i}\left(t ; \mathbb{S}_{t}\right)-\omega_{i, k}, k=1,2, \ldots
$$

Finally, the overall degradation process of PC $i$ subject to piece-wise constant covariates and imperfect PM is given by

$$
\tilde{X}_{i}\left(t ; \mathbb{S}_{t}\right)=\mu_{i} \Lambda\left(\tilde{\omega}_{i}\left(t ; \mathbb{S}_{t}\right)\right)+\xi_{0} B^{(0)}\left(\Lambda\left(\tilde{\omega}_{i}\left(t ; \mathbb{S}_{t}\right)\right)\right)+\xi_{i} B^{(i)}\left(\Lambda\left(\tilde{\omega}_{i}\left(t ; \mathbb{S}_{t}\right)\right)\right)
$$

where $\tilde{\omega}_{i}\left(t ; \mathbb{S}_{t}\right)$ is given by 20 ) or 22 , depending on which imperfect maintenance model is applied.

Figure 8 shows the simulated degradation paths of three PCs with both covariates and imperfect PM effects under the time scale adjustment model. The dotted lines represent the degradation paths involving covariates only (i.e., the same as those red lines in Figure 7]; the red and blue lines represent the degradation paths with both covariates and imperfect maintenance (red: $A R A_{1}$ model; blue: $A R A_{\infty}$ model). One can observe that between two successive PM actions, the degradation paths adjusted by imperfect maintenance are horizontally parallel to the paths without maintenance. That is to say, if we move the red or blue paths to the left, then they would coincide with those dotted lines. This is because an imperfect PM action reduces the current virtual age to an earlier age, and the degradation process then evolves along the same path as that without maintenance. Another observation noteworthy is that in Figure 8 (c) the blue line is even above the red line in some cases, although the corresponding $A R A_{\infty}$ model reduces the virtual age to a lower level than the $A R A_{1}$ model. This is also attributed to the non-monotone property of the Wiener process.

\section{3 | Further discussions}

Analogous to the degradation path adjustment model, the proposed time scale adjustment model can also be reduced to a model with pure covariates or imperfect maintenance effects. More specifically, when $\zeta_{i}\left(s_{j} ; \boldsymbol{\kappa}_{i}\right)=1$ for all $j$, then the proposed model reduces to a model with only imperfect maintenance effects; when $\delta_{i, k}=0$ for all $k$, then there is only time-variant covariate effect; whereas when $\zeta_{i}\left(s_{l} ; \boldsymbol{\kappa}_{i}\right)=1$ and $\delta_{i, k}=0$ for all $j$ and $k$, then the proposed model simplifies to the original model in (2).

In this model, the virtual time scales $\tilde{\omega}_{i}\left(t ; \mathbb{S}_{t}\right)$ of noise processes $B^{(i)}(\Lambda(\cdot)), i=0,1, \ldots, n$, are different due to the influences of covariates and imperfect PM actions. We re-order the $n$ virtual time scales at any $t \in\left[t_{k}, t_{k+1}\right)$ in an ascending order, i.e., $\tilde{\omega}_{(1)}\left(t ; \mathbb{S}_{t}\right) \leq \tilde{\omega}_{(2)}\left(t ; \mathbb{S}_{t}\right) \leq \cdots \leq \tilde{\omega}_{(n)}\left(t ; \mathbb{S}_{t}\right)$, and let $\tilde{X}_{(i)}\left(t ; \mathbb{S}_{t}\right)$ be the overall degradation process corresponding to $\tilde{\omega}_{(i)}\left(t ; \mathbb{S}_{t}\right)$. 
Further define $\Delta \tilde{X}_{(i)}=\tilde{X}_{(i)}\left(t+\Delta t ; \mathbb{S}_{t+\Delta t}\right)-\tilde{X}_{(i)}\left(t ; \mathbb{S}_{t}\right)$ and $\Delta \Lambda_{(i)}=\Lambda\left(\tilde{\omega}_{(i)}\left(t+\Delta t ; \mathbb{S}_{t+\Delta t}\right)\right)-\Lambda\left(\tilde{\omega}_{(i)}\left(t ; \mathbb{S}_{t}\right)\right)$ for any $\Delta t>0$. In some mild conditions, we have the following probabilistic properties on the increments of degradation processes.

Property 2. $\mathrm{E}\left[\Delta \tilde{X}_{(i)}\right]=\mu_{(i)} \Delta \Lambda_{(i)}$, and $\operatorname{Var}\left(\Delta \tilde{X}_{(i)}\right)=\left(\xi_{0}^{2}+\xi_{(i)}^{2}\right) \Delta \Lambda_{(i)}$. If the ascending order of $\tilde{\omega}_{(i)}\left(t ; \mathbb{S}_{t}\right)$ well preserves over $[t, t+\Delta t]$, then for $i \leq i^{\prime}$ we have

$$
\operatorname{Cov}\left(\Delta \tilde{X}_{(i)}, \Delta \tilde{X}_{\left(i^{\prime}\right)}\right)= \begin{cases}0, & \text { if } \tilde{\omega}_{(i)}\left(t+\Delta t ; \mathbb{S}_{t+\Delta t}\right) \leq \tilde{\omega}_{\left(i^{\prime}\right)}\left(t ; \mathbb{S}_{t}\right) ; \\ \xi_{0}^{2}\left[\Lambda\left(\tilde{\omega}_{(i)}\left(t+\Delta t ; \mathbb{S}_{t+\Delta t}\right)\right)-\Lambda\left(\tilde{\omega}_{\left(i^{\prime}\right)}\left(t ; \mathbb{S}_{t}\right)\right)\right], & \text { if } \tilde{\omega}_{(i)}\left(t+\Delta t ; \mathbb{S}_{t+\Delta t}\right)>\tilde{\omega}_{\left(i^{\prime}\right)}\left(t ; \mathbb{S}_{t}\right) .\end{cases}
$$

Based on the results above, the correlation coefficient between any $\Delta \tilde{X}_{(i)}$ and $\Delta \tilde{X}_{\left(i^{\prime}\right)}, i \neq i^{\prime}$, is either $\tilde{\rho}_{(i),\left(i^{\prime}\right)}=0$ or

$$
\tilde{\rho}_{(i),\left(i^{\prime}\right)}=\frac{\xi_{0}^{2}\left[\Lambda\left(\tilde{\omega}_{(i)}\left(t+\Delta t ; \mathbb{S}_{t+\Delta t}\right)\right)-\Lambda\left(\tilde{\omega}_{\left(i^{\prime}\right)}\left(t ; \mathbb{S}_{t}\right)\right)\right]}{\sqrt{\left(\xi_{0}^{2}+\xi_{i}^{2}\right) \Delta \Lambda_{(i)}\left(\xi_{0}^{2}+\xi_{i^{\prime}}^{2}\right) \Delta \Lambda_{\left(i^{\prime}\right)}}},
$$

which is different from $\rho_{i, i^{\prime}}$ in $(3)$ and dependent on time $t$. Property 2 shows that unlike the degradation path adjustment model, the incorporation of covariates and imperfect maintenance effects via the time scale adjustment model changes the dependency structure among individual degradation processes; also, the infinite divisibility property is no longer preserved.

\section{5 | COMPARISONS OF THE PROPOSED MODELS}

In this section, we compare the degradation paths for the degradation path adjustment and time scale adjustment models both analytically and graphically.

We first look at the degradation paths involving only piece-wise constant covariates in (8) and (18). The following result on the mean degradation paths can be obtained.

Property 3. When $\Lambda(t)=t$ and $\mu_{i}\left(s_{j} ; \gamma_{i}\right)=\mu_{i} \cdot \zeta_{i}\left(s_{j} ; \boldsymbol{\kappa}_{i}\right)$ for all $j$, the mean degradation paths $\mathrm{E}\left[X_{i}\left(t ; \mathbb{S}_{t}\right)\right]$ for the degradation path adjustment and time scale adjustment models are identical.

By further examining the overall degradation paths involving both covariates and imperfect maintenance in (10), (12), and 23], we have the following result about the mean degradation paths.

Property 4. When $\Lambda(t)=t, \mu_{i}\left(s_{j} ; \gamma_{i}\right)=\mu_{i} \cdot \zeta_{i}\left(s_{j} ; \boldsymbol{\kappa}_{i}\right)$ for all $j$, and the same $\delta_{i, k}$ for all $k$ are applied, the mean degradation paths $\mathrm{E}\left[\tilde{X}_{i}\left(t ; \mathbb{S}_{t}\right)\right]$ for the degradation path adjustment and time scale adjustment models are identical.

Basically, the validity of Properties 3 and 4 is built upon the linearity of the mean baseline paths in (2). If the baseline processes' mean paths are not linear, then Properties 3 and 4 would no longer hold. Moreover, the actual degradation paths under the two models are not necessarily identical, though the associated mean degradation paths are the same. This is stemmed from the influence of their distinct noise processes.

We then demonstrate and compare the two models through simulated degradation paths. For this purpose, we use the same parameter values (unless specified otherwise) as in Sections 3 and 4 but simulate a new dataset for generating the degradation paths. Figure 9 shows the baseline and adjusted degradation paths of three PCs with time-variant covariates. In this figure, we specify two values for $\beta$, i.e., $\beta=1.0$ and $\beta=1.2$, to represent linear and non-linear mean baseline paths, respectively. The dotted lines represent the baseline degradation paths, whereas the red and blue lines indicate the adjusted degradation paths with covariates, under the degradation path adjustment and time scale adjustment models, respectively. One can see that the adjusted degradation paths under the two models are quite close when the mean baseline path is linear (i.e., $\beta=1.0$ ) and the uncertainty level is low (i.e., for PCs 1 and 2); moreover, the gap between the two paths tends to enlarge as time goes by.

We further demonstrate the overall degradation paths of the three PCs with time-variant covariates and imperfect maintenance effects (see Figure 10 for $A R D_{1}$ and $A R A_{1}$ models; Figure 11 for $A R D_{\infty}$ and $A R A_{\infty}$ ). In addition to $\beta$, we specify two values for the maintenance efficiency $\delta_{i, k}$, i.e., $\delta_{i, k}=0.25$ and $\delta_{i, k}=0.75$, to indicate small and large maintenance efficiencies, respectively. One can observe that the overall degradation paths under the $A R D_{1}$ and $A R A_{1}$ models (as well as the $A R D_{\infty}$ and $A R A_{\infty}$ models) are close when the mean baseline path is linear, the uncertainty level is low, and the maintenance efficiency $\delta_{i, k}$ is small. 

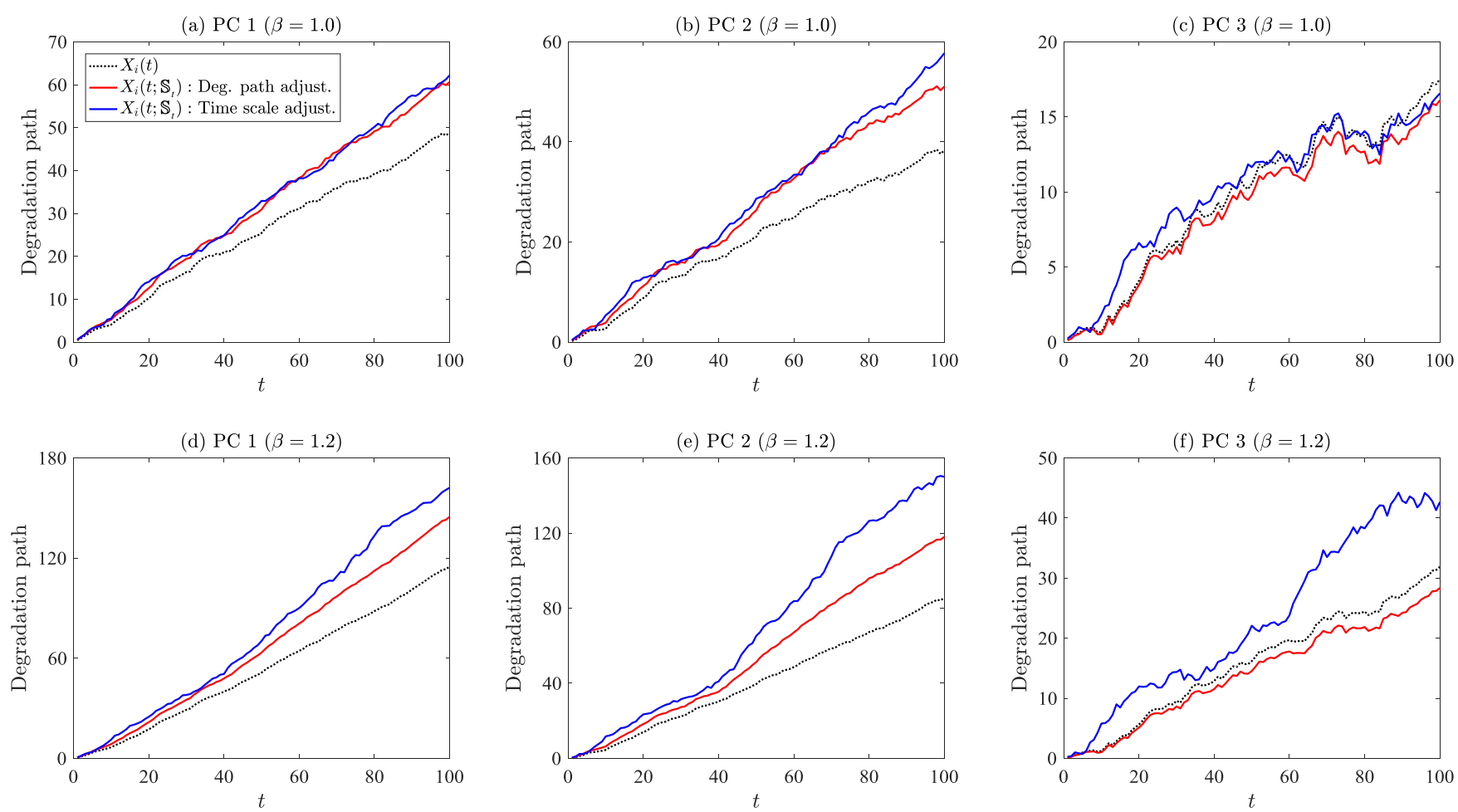

FIGURE 9 Baseline and adjusted degradation paths of three PCs with time-variant covariates for $\beta=1.0$ and $\beta=1.2$, respectively.

\section{6 | CONCLUDING REMARKS}

Degradation and maintenance modeling of complex industrial systems often faces important features such as multiple dependent PCs, time-variant covariates, and imperfect maintenance interventions. This article developed two types of multivariate degradation models subject to time-variant (precisely, piece-wise constant) covariates and imperfect maintenance effects. A multivariate Wiener process was first constructed from multiple univariate Wiener processes sharing a common noise. Then, the degradation path adjustment and time scale adjustment models were developed to unify the modeling of covariates and imperfect maintenance. In particular, two imperfect maintenance models-reduction of degradation level and reduction of virtual age, of both memory one and infinite memory, were proposed. We showed that the proposed models contain some models as special cases; under certain conditions, the proposed two models can generate identical mean degradation paths. However, a drawback of the time scale adjustment model is that when the degradation process is non-monotone, like the Wiener process, the degradation level after maintenance might be even higher than that before maintenance, especially when the variability of the degradation process is high.

Nevertheless, the modeling frameworks developed in this article represent an initial step towards multivariate degradation modeling with time-variant covariates and imperfect maintenance effects. A lot of research work can be done in the next steps, which are briefly discussed below.

(i) Stochastic properties. The stochastic properties of the proposed models, e.g., higher order moments, ergodicity, and asymptotic behaviors, would be of interest and need further research. For example, what are the asymptotic behaviors of the proposed models when $t \rightarrow \infty$. In addition to the simple comparisons in Section 5 in-depth stochastic comparisons of the two modeling frameworks as in Mercier and $\operatorname{Castro}^{7}$ would be another interesting research topic.

(ii) Statistical inference. The complexity of the two modeling frameworks poses challenges for statistical inference. Parameter estimation of the degradation path adjustment model is relatively simple, since the degradation increments between any two successive PM actions follow a multivariate normal distribution (see Property 1 and related discussions). The loglikelihood function can then be formulated using dataset of degradation increments, and various methods, like Bayesian 

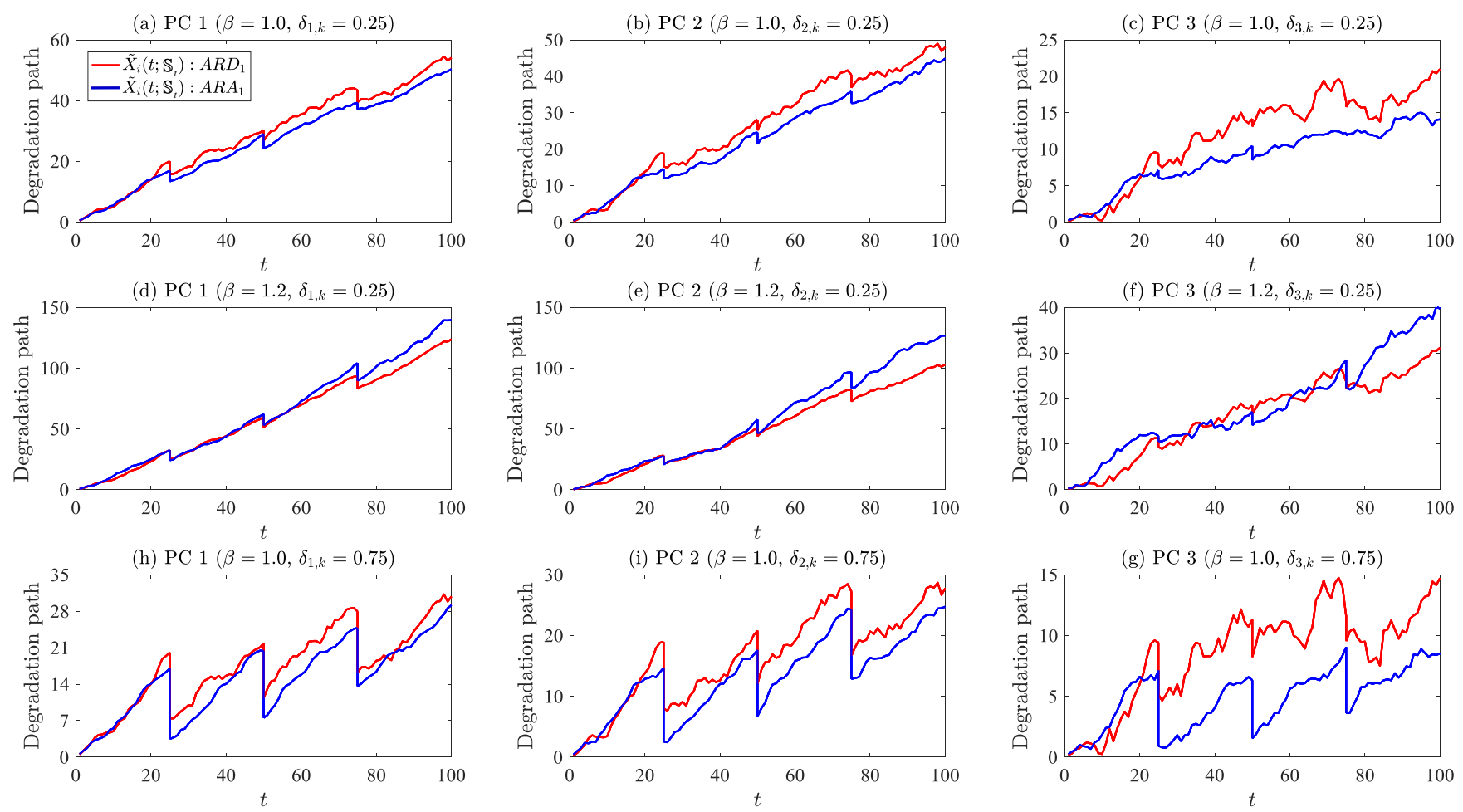

FIGURE 10 Overall degradation paths of three PCs involving time-variant covariates and imperfect PM effects $\left(A R D_{1}\right.$ and $\left.A R A_{1}\right)$.

Markov chain Monte Carlo, can be employed to solve the problem. ${ }^{[14 \mid 15}$ However, parameter estimation of the time scale adjustment model is rather complicated, since the incorporation of covariates and imperfect maintenance alters the dependency structure among individual degradation processes (see Property 2 and related discussions). Additional difficulty arises when the PCs are not always measured at the same time. In addition to the point estimation, interval estimation is usually of more interest in practice, since it quantifies uncertainties in the estimation; the generalized pivots concept in Hong et al. ${ }^{\sqrt{36}}$ might be helpful for interval estimation. Effective statistical tests should also be developed for model discrimination and selection purposes. 64

(iii) Reliability analysis. In accordance with the convention, the failure of a multivariate degrading system can be defined upon threshold $D_{i}$ for each PC $i, i=1,2, \ldots, n$. Specifically, the system is considered to be failed if any of the $n$ PCs exceeds the corresponding failure threshold $D_{i}$. The probability of system failure at time $t$ thus becomes

$$
F(t)=1-\operatorname{Pr}\left\{\tilde{X}_{1}\left(t ; \mathbb{S}_{t}\right)<\mathcal{D}_{1}, \ldots, \tilde{X}_{n}\left(t ; \mathbb{S}_{t}\right)<\mathcal{D}_{n}\right\} .
$$

According to $[25), F(t)$ can be evaluated by integrating $\tilde{X}_{i}\left(t ; \mathbb{S}_{t}\right)$ over $\left(0, \mathcal{D}_{i}\right)$ for all $i=1,2, \ldots, n \cdot \frac{3625}{}$ Again, this is relatively easy for the degradation path adjustment model, but not for the other one. As the closed-form expression of $F(t)$ (or $R(t)=1-F(t)$ ) is difficult to obtain, the semiparametric and nonparametric approximation methods in Palayangoda and $\mathrm{Ng}^{22}$ might be helpful.

(iv) Maintenance planning. Condition-based inspection and maintenance planning for multivariate degrading systems remains under-explored. $\frac{11122}{11}$ is of academic and practical interests to determine the optimal condition-based inspection plan and imperfect PM strategy (maintenance thresholds and degrees) to balance the inspection cost, PM cost, and failure cost; the dependency among individual degradation processes should be well incorporated in this problem. In particular, Markov decision process can be employed to formulate the maintenance planning problem and dynamic programming can be adopted to solve the problem, as in Liu et al. $\frac{\sqrt{45}}{\mathrm{In}} \mathrm{I}$ view of the problem complexity, approximation and simulation techniques might be helpful. 

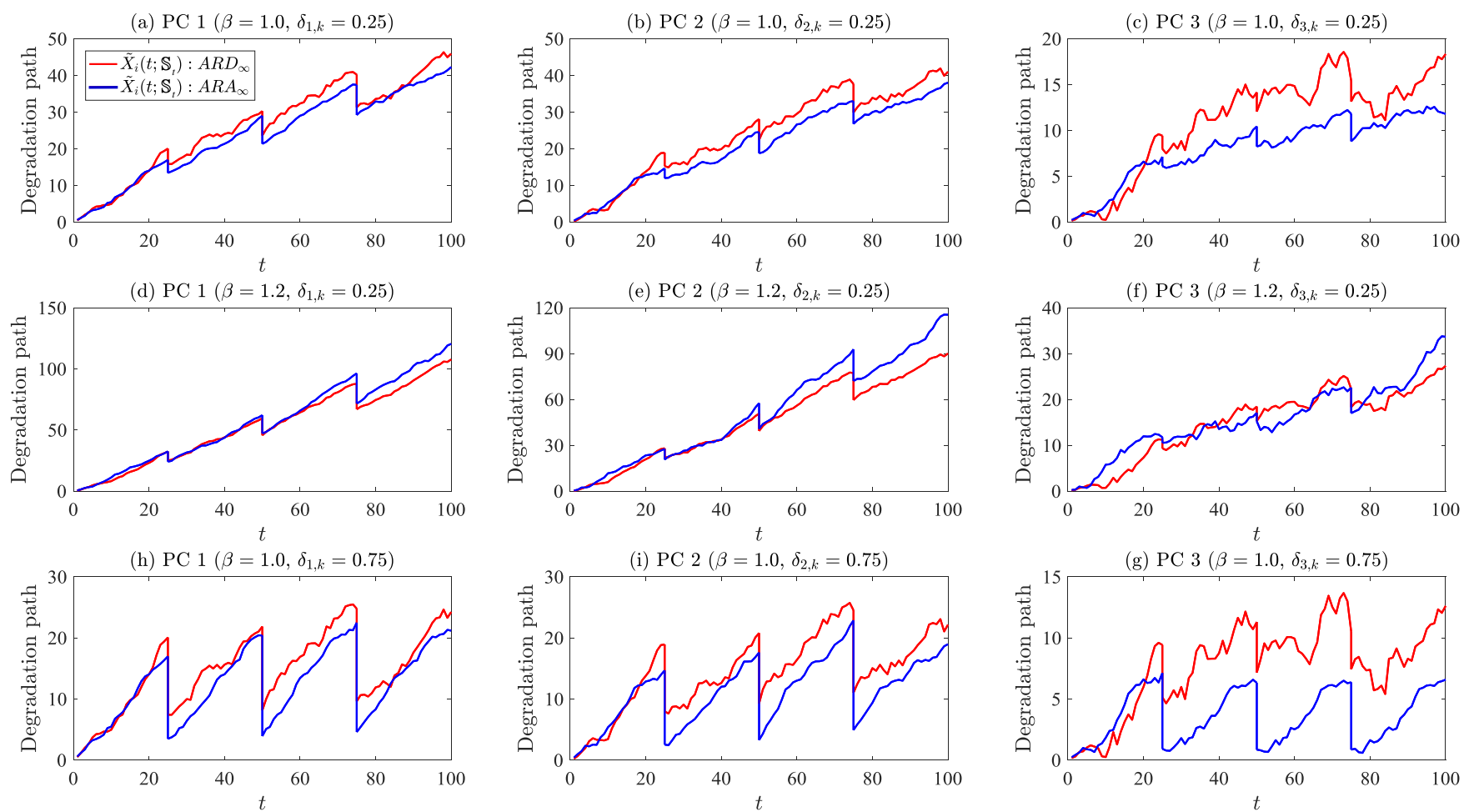

FIGURE 11 Overall degradation paths of the three PCs involving time-variant covariates and imperfect PM effects $\left(A R D_{\infty}\right.$ and $\left.A R A_{\infty}\right)$.

Nevertheless, we hope that this article opens up opportunities for future research in the development of multivariate degradation models wherein the mechanisms of imperfect maintenance and the characteristics of operating/environmental factors play a key role.

\section{ACKNOWLEDGMENTS}

This work was supported by the PHC PROCORE - France/Hong Kong Joint Research Scheme (grant numbers 42636VE, FCityU103/18), the Hong Kong Research Grants Council under a Theme-based Research Scheme (grant number T32-101/15-R) and a General Research Fund (grant number CityU 11203519), and the National Natural Science Foundation of China (grant number 71971181). The work was done while the first author was visiting the Univ. Grenoble Alpes, while at City University of Hong Kong.

\section{DATA AVAILABILITY STATEMENT}

Data sharing is not applicable to this article as no new data were created or analyzed in this study.

\section{References}

1. Doyen Laurent, Gaudoin Olivier. Classes of imperfect repair models based on reduction of failure intensity or virtual age. Reliability Engineering \& System Safety. 2004;84(1):45-56.

2. Wu Shaomin, Zuo Ming J. Linear and nonlinear preventive maintenance models. IEEE Transactions on Reliability. 2010;59(1):242-249. 
3. Ye Zhi-Sheng, Xie Min. Stochastic modelling and analysis of degradation for highly reliable products. Applied Stochastic Models in Business and Industry. 2015;31(1):16-32.

4. Zhang Zhengxin, Si Xiaosheng, Hu Changhua, Lei Yaguo. Degradation data analysis and remaining useful life estimation: A review on Wiener-process-based methods. European Journal of Operational Research. 2018;271(3):775-796.

5. Lu C Joseph, Meeker William Q. Using degradation measures to estimate a time-to-failure distribution. Technometrics. 1993;35(2):161-174.

6. Kahle Waltraud. Imperfect repair in degradation processes: A Kijima-type approach. Applied Stochastic Models in Business and Industry. 2019;35(2):211-220.

7. Mercier Sophie, Castro I T. Stochastic comparisons of imperfect maintenance models for a gamma deteriorating system. European Journal of Operational Research. 2019;273(1):237-248.

8. Singpurwalla Nozer D. Survival in dynamic environments. Statistical Science. 1995;10(1):86-103.

9. Peng Weiwen, Hong Lanqing, Ye Zhisheng. Degradation-based reliability modeling of complex systems in dynamic environments. In: Singapore: Springer 2017 (pp. 81-103).

10. Noortwijk Jan M. A survey of the application of gamma processes in maintenance. Reliability Engineering \& System Safety. 2009;94(1):2-21.

11. Alaswad Suzan, Xiang Yisha. A review on condition-based maintenance optimization models for stochastically deteriorating system. Reliability Engineering \& System Safety. 2017;157:54-63.

12. Jonge Bram, Scarf Philip A. A review on maintenance optimization. European Journal of Operational Research. 2020;285(3):805-824.

13. Sari J K, Newby M J, Brombacher A C, Tang Loon Ching. Bivariate constant stress degradation model: LED lighting system reliability estimation with two-stage modelling. Quality and Reliability Engineering International. 2009;25(8):1067-1084.

14. Pan Zhengqiang, Balakrishnan N, Sun Quan, Zhou Jinglun. Bivariate degradation analysis of products based on Wiener processes and copulas. Journal of Statistical Computation and Simulation. 2013;83(7):1316-1329.

15. Wang Xiaolin, Guo Bo, Cheng Zhijun. Residual life estimation based on bivariate Wiener degradation process with timescale transformations. Journal of Statistical Computation and Simulation. 2014;84(3):545-563.

16. Wang Xiaolin, Balakrishnan N, Guo Bo, Jiang Ping. Residual life estimation based on bivariate non-stationary gamma degradation process. Journal of Statistical Computation and Simulation. 2015;85(2):405-421.

17. Peng Weiwen, Li Yan-Feng, Mi Jinhua, Yu Le, Huang Hong-Zhong. Reliability of complex systems under dynamic conditions: a Bayesian multivariate degradation perspective. Reliability Engineering \& System Safety. 2016;153:75-87.

18. Peng W, Li Y-F, Yang Y-J, Zhu S-P, Huang H-Z. Bivariate analysis of incomplete degradation observations based on inverse Gaussian processes and copulas. IEEE Transactions on Reliability. 2016;65(2):624-639.

19. Peng Weiwen, Ye Zhi-Sheng, Chen Nan. Joint online RUL prediction for multivariate deteriorating systems. IEEE Transactions on Industrial Informatics. 2019;15(5):2870-2878.

20. Fang Guanqi, Pan Rong, Hong Yili. Copula-based reliability analysis of degrading systems with dependent failures. Reliability Engineering \& System Safety. 2020;193:106618.

21. Liu Bin, Zhao Xiujie, Liu Guoquan, Liu Yiqi. Life cycle cost analysis considering multiple dependent degradation processes and environmental influence. Reliability Engineering \& System Safety. 2020;197:106784.

22. Palayangoda Lochana K, Ng Hon Keung Tony. Semiparametric and nonparametric evaluation of first-passage distribution of bivariate degradation processes. Reliability Engineering \& System Safety. 2021;205:107230. 
23. Hong Yili, Zhang Man, Meeker William Q. Big data and reliability applications: The complexity dimension. Journal of Quality Technology. 2018;50(2):135-149.

24. Si Wujun, Yang Qingyu, Wu Xin, Chen Yong. Reliability analysis considering dynamic material local deformation. Journal of Quality Technology. 2018;50(2):183-197.

25. Lu Lu, Wang Bing Xing, Hong Yili, Ye Zhisheng. General path models for degradation data with multiple characteristics and covariates. Technometrics. 2020;:DOI: 10.1080/00401706.2020.1796814.

26. Whitmore G A, Crowder M J, Lawless J F. Failure inference from a marker process based on a bivariate Wiener model. Lifetime Data Analysis. 1998;4(3):229-251.

27. Lee Mel-Ling Ting, DeGruttola Victor, Schoenfeld David. A model for markers and latent health status. Journal of the Royal Statistical Society: Series B (Statistical Methodology). 2000;62(4):747-762.

28. Liu Xiao, Al-Khalifa Khalifa N, Elsayed Elsayed A, Coit David W, Hamouda Abdelmagid S. Criticality measures for components with multi-dimensional degradation. IIE Transactions. 2014;46(10):987-998.

29. $\mathrm{Xu}$ Ancha, Shen Lijuan, Wang Bingxing, Tang Yincai. On modeling bivariate Wiener degradation process. IEEE Transactions on Reliability. 2018;67(3):897-906.

30. Dong Qinglai, Cui Lirong, Si Shubin. Reliability and availability analysis of stochastic degradation systems based on bivariate Wiener processes. Applied Mathematical Modelling. 2020;79:414-433.

31. Hajiha Mohammadmahdi, Liu Xiao, Hong Yili. Degradation under dynamic operating conditions: Modeling, competing processes and applications. Journal of Quality Technology. 2020;:DOI: 10.1080/00224065.2020.1757390.

32. Pan Zhengqiang, Balakrishnan N. Reliability modeling of degradation of products with multiple performance characteristics based on gamma processes. Reliability Engineering \& System Safety. 2011;96(8):949-957.

33. Pan Zhengqiang, Sun Quan. Optimal design for step-stress accelerated degradation test with multiple performance characteristics based on gamma processes. Communications in Statistics-Simulation and Computation. 2014;43(2):298-314.

34. Mercier Sophie, Pham Hai Ha. A preventive maintenance policy for a continuously monitored system with correlated wear indicators. European Journal of Operational Research. 2012;222(2):263-272.

35. Mercier Sophie, Meier-Hirmer Carolina, Roussignol Michel. Bivariate Gamma wear processes for track geometry modelling, with application to intervention scheduling. Structure and Infrastructure Engineering. 2012;8(4):357-366.

36. Hong Lanqing, Ye Zhi-Sheng, Ling Ran. Environmental risk assessment of emerging contaminants using degradation data. Journal of Agricultural, Biological and Environmental Statistics. 2018;23(3):390-409.

37. Sun Qiuzhuang, Ye Zhi-Sheng, Hong Yili. Statistical modeling of multivariate destructive degradation tests with blocking. Technometrics. 2020;62(4):536-548.

38. Sun Qiuzhuang, Ye Zhi-Sheng, Zhu Xiaoyan. Managing component degradation in series systems for balancing degradation through reallocation and maintenance. IISE Transactions. 2020;52(7):797-810.

39. Iyengar Satish. Hitting lines with two-dimensional Brownian motion. SIAM Journal on Applied Mathematics. 1985;45(6):983-989.

40. Barker C T, Newby M J. Optimal non-periodic inspection for a multivariate degradation model. Reliability Engineering \& System Safety. 2009;94(1):33-43.

41. Ahmadi R. An optimal replacement policy for complex multi-component systems. International Journal of Production Research. 2016;54(17):5303-5316.

42. Wu Shaomin, Castro Inma T. Maintenance policy for a system with a weighted linear combination of degradation processes. European Journal of Operational Research. 2020;280(1):124-133. 
43. Castanier B, Grall A, Bérenguer C. A condition-based maintenance policy with non-periodic inspections for a two-unit series system. Reliability Engineering \& System Safety. 2005;87(1):109-120.

44. Li Heping, Deloux Estelle, Dieulle Laurence. A condition-based maintenance policy for multi-component systems with Lévy copulas dependence. Reliability Engineering \& System Safety. 2016;149:44-55.

45. Liu Bin, Pandey Mahesh D., Wang Xiaolin, Zhao Xiujie. A finite-horizon condition-based maintenance for a two-unit system with dependent degradation processes. European Journal of Operational Research. 2021;:Submitted for publication.

46. Castanier B, Bérenguer C, Grall A. A sequential condition-based repair/replacement policy with non-periodic inspections for a system subject to continuous wear. Applied Stochastic Models in Business and Industry. 2003;19(4):327-347.

47. Mercier Sophie, Castro IT. On the modelling of imperfect repairs for a continuously monitored gamma wear process through age reduction. Journal of Applied Probability. 2013;50(4):1057-1076.

48. Zhang Mimi, Gaudoin Olivier, Xie Min. Degradation-based maintenance decision using stochastic filtering for systems under imperfect maintenance. European Journal of Operational Research. 2015;245(2):531-541.

49. Zhao Xiujie, Gaudoin Olivier, Doyen Laurent, Xie Min. Optimal inspection and replacement policy based on experimental degradation data with covariates. IISE Transactions. 2019;51(3):322-336.

50. Salles Gabriel, Mercier Sophie, Bordes Laurent. Semiparametric estimate of the efficiency of imperfect maintenance actions for a gamma deteriorating system. Journal of Statistical Planning and Inference. 2020;206:278-297.

51. Meeker W Q, Hong Y. Reliability meets big data: opportunities and challenges. Quality Engineering. 2014;26(1):102-116.

52. Hong Yili, Duan Yuanyuan, Meeker William Q, Stanley Deborah L, Gu Xiaohong. Statistical methods for degradation data with dynamic covariates information and an application to outdoor weathering data. Technometrics. 2015;57(2):180-193.

53. Xu Zhibing, Hong Yili, Jin Ran. Nonlinear general path models for degradation data with dynamic covariates. Applied Stochastic Models in Business and Industry. 2016;32(2):153-167.

54. Tseng Sheng-Tsaing, Wen Zhi-Chih. Step-stress accelerated degradation analysis for highly reliable products. Journal of Quality Technology. 2000;32(3):209-216.

55. Liao Chen-Mao, Tseng Sheng-Tsaing. Optimal design for step-stress accelerated degradation tests. IEEE Transactions on Reliability. 2006;55(1):59-66.

56. Peng Chien-Yu, Tseng Sheng-Tsaing. Progressive-stress accelerated degradation test for highly-reliable products. IEEE Transactions on Reliability. 2010;59(1):30-37.

57. Zhao Xiujie, Pan Rong, Xie Min. Bayesian planning of step-stress accelerated degradation tests under various optimality criteria. Applied Stochastic Models in Business and Industry. 2019;35(3):537-551.

58. Lim Heonsang, Yum Bong-Jin. Optimal design of accelerated degradation tests based on Wiener process models. Journal of Applied Statistics. 2011;38(2):309-325.

59. Zhao Xiujie, He Kangzhe, Kuo Way, Xie Min. Planning accelerated reliability tests for mission-oriented systems subject to degradation and shocks. IISE Transactions. 2020;52(1):91-103.

60. Kahle Waltraud, Mercier Sophie, Paroissin Christian. Degradation Processes in Reliability. London, UK: John Wiley \& Sons; 2016.

61. Nelson Wayne B. Accelerated Testing: Statistical Models, Test Plans, and Data Analysis. New Jersey, USA: John Wiley \& Sons; 1990.

62. Finkelstein Maxim, Cha Ji Hwan. Virtual age, is it real?-Discussing virtual age in reliability context. Applied Stochastic Models in Business and Industry. 2020;:DOI: 10.1002/asmb.2567.

63. Kijima M. Some results for repairable systems with general repair. Journal of Applied Probability. 1989;26(1):89-102. 
64. Chen Piao, Ye Zhi-Sheng, Xiao Xun. Pairwise model discrimination with applications in lifetime distributions and degradation processes. Naval Research Logistics (NRL). 2019;66(8):675-686.

\section{APPENDIX}

Proof of Property 1$]$ From (10) and (12), we know that $\Delta \tilde{X}_{i}=\tilde{X}_{i}\left(t+\Delta t ; \mathbb{S}_{t+\Delta t}\right)-\tilde{X}_{i}\left(t ; \mathbb{S}_{t}\right)=X_{i}\left(t+\Delta t ; \mathbb{S}_{t+\Delta t}\right)-X_{i}\left(t ; \mathbb{S}_{t}\right)$ for any $t_{k} \leq t<t+\Delta t<t_{k+1}$, where $X_{i}\left(t ; \mathbb{S}_{t}\right)$ is given by $[8]$. Then, it is clear that $\mathrm{E}\left[\Delta \tilde{X}_{i}\right]=\Delta d_{i}, \operatorname{Var}\left(\Delta \tilde{X}_{i}\right)=\left(\xi_{0}^{2}+\xi_{i}^{2}\right) \Delta \Lambda$, and $\operatorname{Cov}\left(\Delta \tilde{X}_{i}, \Delta \tilde{X}_{i^{\prime}}\right)=\xi_{0}^{2} \Delta \Lambda$ for $i \neq i^{\prime}$.

Proof of Property 2 The mean and variance of $\Delta \tilde{X}_{(i)}$ are straightforward to derive. For the covariance, we have

$$
\begin{aligned}
\operatorname{Cov}\left(\Delta \tilde{X}_{(i)}, \Delta \tilde{X}_{\left(i^{\prime}\right)}\right)= & \operatorname{Cov}\left(\tilde{X}_{(i)}\left(t+\Delta t ; \mathbb{S}_{t+\Delta t}\right)-\tilde{X}_{(i)}\left(t ; \mathbb{S}_{t}\right), \tilde{X}_{\left(i^{\prime}\right)}\left(t+\Delta t ; \mathbb{S}_{t+\Delta t}\right)-\tilde{X}_{\left(i^{\prime}\right)}\left(t ; \mathbb{S}_{t}\right)\right) \\
= & \operatorname{Cov}\left(\tilde{X}_{(i)}\left(t+\Delta t ; \mathbb{S}_{t+\Delta t}\right), \tilde{X}_{\left(i^{\prime}\right)}\left(t+\Delta t ; \mathbb{S}_{t+\Delta t}\right)\right)-\operatorname{Cov}\left(\tilde{X}_{(i)}\left(t+\Delta t ; \mathbb{S}_{t+\Delta t}\right), \tilde{X}_{\left(i^{\prime}\right)}\left(t ; \mathbb{S}_{t}\right)\right) \\
& -\operatorname{Cov}\left(\tilde{X}_{(i)}\left(t ; \mathbb{S}_{t}\right), \tilde{X}_{\left(i^{\prime}\right)}\left(t+\Delta t ; \mathbb{S}_{t+\Delta t}\right)\right)+\operatorname{Cov}\left(\tilde{X}_{(i)}\left(t ; \mathbb{S}_{t}\right), \tilde{X}_{\left(i^{\prime}\right)}\left(t ; \mathbb{S}_{t}\right)\right) .
\end{aligned}
$$

For $i \leq i^{\prime}$, we have $\tilde{\omega}_{(i)}\left(t ; \mathbb{S}_{t}\right) \leq \tilde{\omega}_{\left(i^{\prime}\right)}\left(t ; \mathbb{S}_{t}\right)$, and $\Lambda\left(\tilde{\omega}_{(i)}\left(t ; \mathbb{S}_{t}\right)\right) \leq \Lambda\left(\tilde{\omega}_{\left(i^{\prime}\right)}\left(t ; \mathbb{S}_{t}\right)\right)$ as $\Lambda(\cdot)$ is an increasing function. Then, the fourth term in the equation above can be further derived as

$$
\begin{aligned}
\operatorname{Cov}\left(\tilde{X}_{(i)}\left(t ; \mathbb{S}_{t}\right), \tilde{X}_{\left(i^{\prime}\right)}\left(t ; \mathbb{S}_{t}\right)\right) & =\xi_{0}^{2} \operatorname{Cov}\left(B^{(0)}\left(\Lambda\left(\tilde{\omega}_{(i)}\left(t ; \mathbb{S}_{t}\right)\right)\right), B^{(0)}\left(\Lambda\left(\tilde{\omega}_{\left(i^{\prime}\right)}\left(t ; \mathbb{S}_{t}\right)\right)\right)\right) \\
& =\xi_{0}^{2} \Lambda\left(\tilde{\omega}_{(i)}\left(t ; \mathbb{S}_{t}\right)\right) .
\end{aligned}
$$

The second equality holds because for a standard Brownian motion $B(\cdot)$, we have $\operatorname{Cov}(B(s), B(t))=s$ if $s \leq t$.

Likewise, we can easily obtain $\operatorname{Cov}\left(\tilde{X}_{(i)}\left(t ; \mathbb{S}_{t}\right), \tilde{X}_{\left(i^{\prime}\right)}\left(t+\Delta t ; \mathbb{S}_{t+\Delta t}\right)\right)=\xi_{0}^{2} \Lambda\left(\tilde{\omega}_{(i)}\left(t ; \mathbb{S}_{t}\right)\right)$. Moreover, if the order of $\tilde{\omega}_{(i)}\left(t ; \mathbb{S}_{t}\right)$ well preserves over $[t, t+\Delta t]$, i.e., $\tilde{\omega}_{(i)}\left(t+\Delta t ; \mathbb{S}_{t+\Delta t}\right)<\tilde{\omega}_{\left(i^{\prime}\right)}\left(t+\Delta t ; \mathbb{S}_{t+\Delta t}\right)$ for $i<i^{\prime}$, then $\operatorname{Cov}\left(\tilde{X}_{(i)}\left(t+\Delta t ; \mathbb{S}_{t+\Delta t}\right), \tilde{X}_{\left(i^{\prime}\right)}\left(t+\Delta t ; \mathbb{S}_{t+\Delta t}\right)\right)=$ $\xi_{0}^{2} \Lambda\left(\tilde{\omega}_{(i)}\left(t+\Delta t ; \mathbb{S}_{t+\Delta t}\right)\right)$. However, for $\operatorname{Cov}\left(\tilde{X}_{(i)}\left(t+\Delta t ; \mathbb{S}_{t+\Delta t}\right), \tilde{X}_{\left(i^{\prime}\right)}\left(t ; \mathbb{S}_{t}\right)\right)$, we need to consider the following two cases:

(i) If $\tilde{\omega}_{(i)}\left(t+\Delta t ; \mathbb{S}_{t+\Delta t}\right) \leq \tilde{\omega}_{\left(i^{\prime}\right)}\left(t ; \mathbb{S}_{t}\right)$ then $\operatorname{Cov}\left(\tilde{X}_{(i)}\left(t+\Delta t ; \mathbb{S}_{t+\Delta t}\right), \tilde{X}_{\left(i^{\prime}\right)}\left(t ; \mathbb{S}_{t}\right)\right)=\xi_{0}^{2} \Lambda\left(\tilde{\omega}_{(i)}\left(t+\Delta t ; \mathbb{S}_{t+\Delta t}\right)\right)$;

(ii) If $\tilde{\omega}_{(i)}\left(t+\Delta t ; \mathbb{S}_{t+\Delta t}\right)>\tilde{\omega}_{\left(i^{\prime}\right)}\left(t ; \mathbb{S}_{t}\right)$ then $\operatorname{Cov}\left(\tilde{X}_{(i)}\left(t+\Delta t ; \mathbb{S}_{t+\Delta t}\right), \tilde{X}_{\left(i^{\prime}\right)}\left(t ; \mathbb{S}_{t}\right)\right)=\xi_{0}^{2} \Lambda\left(\tilde{\omega}_{\left(i^{\prime}\right)}\left(t ; \mathbb{S}_{t}\right)\right)$.

In the former case, $\operatorname{Cov}\left(\Delta \tilde{X}_{(i)}, \Delta \tilde{X}_{\left(i^{\prime}\right)}\right)=0$, whereas in the latter case, $\operatorname{Cov}\left(\Delta \tilde{X}_{(i)}, \Delta \tilde{X}_{\left(i^{\prime}\right)}\right)=\xi_{0}^{2}\left[\Lambda\left(\tilde{\omega}_{(i)}\left(t+\Delta t ; \mathbb{S}_{t+\Delta t}\right)\right)-\right.$ $\left.\Lambda\left(\tilde{\omega}_{\left(i^{\prime}\right)}\left(t ; \mathbb{S}_{t}\right)\right)\right]$. This completes the proof.

Proof of Property 3 From (8), we know that when $\Lambda(t)=t$, the mean of $X_{i}\left(t ; \mathbb{S}_{t}\right)$ in the degradation path adjustment model is $\mathrm{E}\left[X_{i}\left(t ; \mathbb{S}_{t}\right)\right]=d_{i}\left(t ; \mathbb{S}_{t}\right)=\mu_{i}\left(s_{j} ; \gamma_{i}\right)\left(t-\tau_{j-1}\right)+\sum_{l=1}^{j-1} \mu_{i}\left(s_{l} ; \gamma_{i}\right)\left(\tau_{l}-\tau_{l-1}\right)$ for any $t \in\left[\tau_{j-1}, \tau_{j}\right)$. Similarly, in the time scale adjustment model, the mean of $X_{i}\left(t ; \mathbb{S}_{t}\right)$ in $[18]$ is given by $\mathrm{E}\left[X_{i}\left(t ; \mathbb{S}_{t}\right)\right]=\mu_{i} \omega_{i}\left(t ; \mathbb{S}_{t}\right)=\mu_{i} \zeta_{i}\left(\boldsymbol{s}_{j} ; \boldsymbol{\kappa}_{i}\right)\left(t-\tau_{j-1}\right)+\sum_{l=2}^{j} \mu_{i} \zeta_{i}\left(\boldsymbol{s}_{l-1} ; \boldsymbol{\kappa}_{i}\right)\left(\tau_{l-1}-\right.$ $\left.\tau_{l-2}\right)$ for any $t \in\left[\tau_{j-1}, \tau_{j}\right)$. It is clear that when $\Lambda(t)=t$ and $\mu_{i}\left(\boldsymbol{s}_{j} ; \gamma_{i}\right)=\mu_{i} \cdot \zeta_{i}\left(\boldsymbol{s}_{j} ; \boldsymbol{\kappa}_{i}\right)$ for all $j$, we have $d_{i}\left(t ; \mathbb{S}_{t}\right)=\mu_{i} \omega_{i}\left(t ; \mathbb{S}_{t}\right)$ and thus the two expressions of $\mathrm{E}\left[X_{i}\left(t ; \mathbb{S}_{t}\right)\right]$ are identical.

Proof of Property 4 We first examine the $A R D_{1}$ and $A R A_{1}$ models. Recall that $d_{i, k}=d_{i}\left(t_{k} ; \mathbb{S}_{t_{k}}\right.$ ). According to [10], it is known that the mean of $\tilde{X}_{i}\left(t ; \mathbb{S}_{t}\right)$ in the degradation path adjustment model is $\mathrm{E}\left[\tilde{X}_{i}\left(t ; \mathbb{S}_{t}\right)\right]=\sum_{l=1}^{k}\left(1-\delta_{i, l}\right)\left(d_{i, l}-d_{i, l-1}\right)+d_{i}\left(t ; \mathbb{S}_{t}\right)-d_{i, k}$ for any $t \in\left[t_{k}, t_{k+1}\right)$; in the time scale adjustment model, the mean of $\tilde{X}_{i}\left(t ; \mathbb{S}_{t}\right)$ in $[23]$ is given by $\mathrm{E}\left[\tilde{X}_{i}\left(t ; \mathbb{S}_{t}\right)\right]=\sum_{l=1}^{k}(1-$ $\left.\delta_{i, l}\right) \mu_{i}\left(\omega_{i, l}-\omega_{i, l-1}\right)+\mu_{i}\left(\omega_{i}\left(t ; \mathbb{S}_{t}\right)-\omega_{i, k}\right)$ for any $t \in\left[t_{k}, t_{k+1}\right)$. In Property 3 . we know that $d_{i}\left(t ; \mathbb{S}_{t}\right)=\mu_{i} \omega_{i}\left(t ; \mathbb{S}_{t}\right)$ for any $t \in\left[t_{k}, t_{k+1}\right)$. Combining the condition that the same $\delta_{i, k}$ for all $k$ are applied, the expressions of $\mathrm{E}\left[\tilde{X}_{i}\left(t ; \mathbb{S}_{t}\right)\right]$ for the $A R D_{1}$ and $A R A_{1}$ models are exactly the same.

The case for the $A R D_{\infty}$ and $A R A_{\infty}$ models is very similar and thus omitted. 